\title{
Linear Simulations of the Cylindrical Richtmyer-Meshkov Instability in Hydrodynamics and MHD
}

\author{
Thesis by \\ Song Gao
}

In Partial Fulfillment of the Requirements

For the Degree of

Masters of Science

King Abdullah University of Science and Technology, Thuwal,

Kingdom of Saudi Arabia

May, 2013 
The thesis of Song Gao is approved by the examination committee

Committee Chairperson: Prof. Ravi Samtaney

Committee Member: Prof. Sigurdur Thoroddsen

Committee Member: Prof. Georgiy Stenchikov 
Copyright (C)2013

Song Gao

All Rights Reserved 


\title{
ABSTRACT
}

\section{Linear Simulations of the Cylindrical Richtmyer-Meshkov Instability in Hydrodynamics and MHD}

\author{
Song Gao
}

The Richtmyer-Meshkov instability occurs when density-stratified interfaces are impulsively accelerated, typically by a shock wave. We present a numerical method to simulate the Richtmyer-Meshkov instability in cylindrical geometry. The ideal MHD equations are linearized about a time-dependent base state to yield linear partial differential equations governing the perturbed quantities. Convergence tests demonstrate that second order accuracy is achieved for smooth flows, and the order of accuracy is between first and second order for flows with discontinuities.

Numerical results are presented for cases of interfaces with positive Atwood number and purely azimuthal perturbations. In hydrodynamics, the Richtmyer-Meshkov instability growth of perturbations is followed by a Rayleigh-Taylor growth phase. In MHD, numerical results indicate that the perturbations can be suppressed for sufficiently large perturbation wavenumbers and magnetic fields. 


\section{ACKNOWLEDGEMENTS}

Sincere thanks to my supervisor, Prof. Ravi Samtaney, for his profound Knowledge, patience and enthusiasm.

Thanks to the members of my thesis examination committee, Prof. Sigurdur Thoroddsen and Prof. Georgiy Stenchikov, for their valuable comments and precious time.

Thanks to Dr. Manuel Lombardini, for the interesting discussion and invaluable help. Thanks to $\mathcal{M r}$. Wei Gao, for his great encouragement.

Thanks to my parents, for raising me up, and my unmet wife. 


\section{TABLE OF CONTENTS}

Examination Committee Approval 2

\begin{tabular}{|cc}
\hline Copyright & 3 \\
\hline
\end{tabular}

\begin{tabular}{ll}
\hline Abstract & 4
\end{tabular}

\begin{tabular}{|l|l|}
\hline Acknowledgements & 5
\end{tabular}

\begin{tabular}{lr}
\hline List of Abbreviations & 8
\end{tabular}

\begin{tabular}{ll}
\hline List of Symbols & 9
\end{tabular}

\begin{tabular}{ll}
\hline List of Figures & 11
\end{tabular}

\begin{tabular}{ll}
\hline List of Tables & 14
\end{tabular}

$\begin{array}{lll}1 & \text { Introduction } & 15\end{array}$

$\begin{array}{lll}2 & \text { Governing Equations } & 19\end{array}$

2.1 The Ideal MHD Equations . . . . . . . . . . . . . . . . . . . . . . . . 19

2.2 Linearization of the Ideal MHD Equations . . . . . . . . . . . . . . . 21

2.3 Scale free property of the Ideal Magnetohydrodynamics (MHD) Equations . . . . . . . . . . . . . . . . . . . . 23

$\begin{array}{lll}3 & \text { Numerical Method } & 25\end{array}$

3.1 Temporal Discretization . . . . . . . . . . . . . . . . . . 25

3.2 Spatial Discretization . . . . . . . . . . . . . . . . . . . 26

3.3 Additional Stabilization Term . . . . . . . . . . . . . . . . . . 26

3.4 Seven Wave Method . . . . . . . . . . . . . . . . . . . . . . . . . . . 28

3.4 .1 Numerical flux . . . . . . . . . . . . . . . . . . . . . . . . . . 28

3.4 .2 Preserving the Solenoidal Property of the Magnetic Field . . . 30

3.5 Passive Scalar to Track Density Interface . . . . . . . . . . . . . . . . 31 
3.6 Convergence test $\ldots \ldots \ldots \ldots$

3.6 .1 Numerical convergence test . . . . . . . . . . . . . . . 32

3.6 .2 Convergence test for a single shocked interface . . . . . . . 38

4 Linear Stability Results of Richtmyer-Meshkov instability (RMI) in $\begin{array}{ll}\text { Cylindrical Coordinates } & 41\end{array}$

4.1 Parameters of Investigation $\ldots \ldots \ldots \ldots$

4.2 Solution Details of Base State . . . . . . . . . . . . . . . . 42

4.3 Solution Details for RMI in Hydrodynamics $\ldots \ldots \ldots \ldots$

4.4 Solution Details for RMI in MHD . . . . . . . . . . . . . . 50

4.5 Comparison between hydrodynamics and MHD $\ldots \ldots \ldots \ldots . . \ldots 53$

$\begin{array}{lll}5 \text { Conclusion } & 57\end{array}$

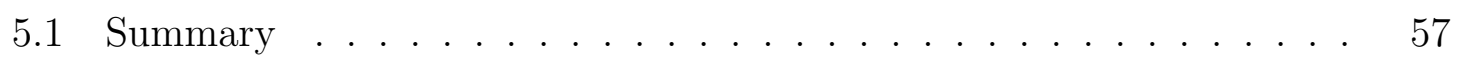

5.2 Future Research Work $\ldots \ldots \ldots \ldots \ldots$

\begin{tabular}{ll}
\hline References & 59
\end{tabular}

\begin{tabular}{ll}
\hline Appendices & 61
\end{tabular}

A.1 Governing equations of Chisnell-type shock . . . . . . . . . . . 62

A.2 Determining self-similar coefficient $\alpha \ldots \ldots \ldots \ldots \ldots$ 


\title{
LIST OF ABBREVIATIONS
}

\author{
AF Alfvén front \\ CD contact discontinuity \\ ICF inertial confinement fusion \\ IS incident shock \\ MHD Magnetohydrodynamics \\ RHS right hand side \\ RMI Richtmyer-Meshkov instability \\ RS reflected shock \\ RTI Rayleigh-Taylor instability \\ TS transmitted shock \\ TVD total variation diminishing \\ VS vortex sheet
}




\section{LIST OF SYMBOLS}

A Atwood number

$B_{r} \quad r$-component of magnetic field

$B_{\theta} \quad \theta$-component of magnetic field

$B_{z} \quad z$-component of magnetic field

$\beta \quad$ plasma beta

$\Delta r \quad$ spatial mesh size in radial direction

$\Delta t \quad$ time step

$e \quad$ total energy per unit volume

$F(U) \quad$ r-component of flux

$G(U) \quad \theta$-component of flux

$\gamma \quad$ ratio of specific heats

$\dot{h}$ perturbation growth rate at the interface

$i \quad$ index of mesh interval

j imaginary number $\sqrt{-1}$

$A\left(\dot{U}^{\circ}\right) \quad$ Jacobian matrix of flux $F\left(\dot{U}^{\circ}\right)$ with respect to $\stackrel{\circ}{U}$

$M(\stackrel{\circ}{U}) \quad$ Jacobian matrix of flux $G(\stackrel{\circ}{U})$ with respect to $\stackrel{\circ}{U}$

$\bar{A}(\stackrel{\circ}{U}) \quad$ matrix $A(\stackrel{\circ}{U})$ excluding the 5-th row and 5-th column 
$\bar{M}(\stackrel{\circ}{U})$ matrix $M(\stackrel{\circ}{\dot{U}})$ excluding the 5-th row and 5-th column

$m \quad$ azimuthal perturbation wavenumber

$M_{s} \quad$ Mach number of shock

$p \quad$ gas pressure

$\tilde{p} \quad$ total pressure

$\psi \quad$ passive scale to track density interface

$R_{0} \quad$ radial coordinate of interface

$R_{l} \quad$ radial coordinate of left boundary

$R_{r} \quad$ radial coordinate of right boundary

$R_{s} \quad$ radial coordinate of shock

$\rho \quad$ density

$t \quad$ time

U solution vector

$\stackrel{\circ}{U} \quad$ solution vector of base state

$\hat{U} \quad$ solution vector of perturbed state

$\stackrel{\overline{0}}{U} \quad$ solution vector of base state $\stackrel{\circ}{U}$ excluding the 5-th row

$\overline{\hat{U}} \quad$ solution vector of perturbed state $\hat{U}$ excluding the 5 -th row

$u_{r} \quad r$-component of velocity field

$u_{\theta} \quad \theta$-component of velocity field

$u_{z} \quad z$-component of velocity field 


\section{LIST OF FIGURES}

$1.1 \quad$ Illustration of the physical setup. A perturbed interface is located at $r=R_{0}$, separating two fluids of density $\rho_{1}$ and $\rho_{2}$. An incident shock is located at $r=R_{s}$, with Mach number $M_{s}$. The whole domain is bounded by left boundary and right boundary. . . . . . . . . . . . . . 18

$3.1 \quad$ Illustration of spatial discretization. Circles are the centroids of each sub-intervals. The solution vectors are only defined at centroids. Fluxes are computed on two neighboring edges. . . . . . . . . . . . . . . 26

$3.2 \quad$ Illustration of passive scalar $\psi$ to track density interface. The interface

is located at $R_{0}=1.0$. . . . . . . . . . . . . . . . . . 32

3.3 The initial profile of perturbed density $\hat{\rho}$ at time $t=0.00$ for numerical convergence test. . . . . . . . . . . . . . . . . 33

$3.4 \quad$ For hydrodynamics, the profiles of (a) perturbed $r$-component of velocity $\hat{u}_{r}(\mathrm{~b})$ perturbed $r$-component of momentum $\hat{\rho} \hat{u}_{r}(\mathrm{c})$ perturbed $\theta$-component of momentum $\hat{\rho} u_{\theta}(\mathrm{d})$ perturbed energy density $\hat{e}$ at time $t=0.12$ for mesh size 400, 800, 1600, 3200 and 6400 . . . . . . . . . . 34

3.5 For MHD, the profiles of (a) perturbed $r$-component of velocity $\hat{u}_{r}(\mathrm{~b})$ perturbed $r$-component of momentum $\hat{\rho} \hat{u}_{r}(\mathrm{c})$ perturbed $\theta$-component of momentum $\hat{\rho} u_{\theta}(\mathrm{d})$ perturbed energy density $\hat{e}(\mathrm{e})$ perturbed $r$ component of magnetic field $\hat{B}_{r}(\mathrm{f})$ perturbed $\theta$-component of magnetic field $\hat{B}_{\theta}$ at time $t=0.12$ for mesh size 400, 800, 1600,3200 and 6400. 37

$3.6 \quad$ The initial profile of perturbed density $\hat{\rho}$ at time $t=0$ for a single shocked interface case. Profile is an approximation of a delta function 38

3.7 Time history of unscaled growth rate for the case of a single shocked interface using different mesh size 800, 1600, 3200, 6400, 12800. . . . 39

3.8 Convergence plot of unscaled growth rate for the case of a single shocked interface at time $t=0.288$. . . . . . . . . . . . . . . . 40

4.1 The initial profile of perturbed density $\hat{\rho}$ at time $t=0$ for a single shocked interface case. Profile is an approximation of a delta function 42 
4.12 Profiles of (a) perturbed z-component of vorticity for hydrodynamics. (f) perturbed z-component of vorticity for MHD. The perturbation wavenumber is $m=256$ and the plasma beta is $\beta=4$ for MHD. . . . 54

4.13 (a) Spacetime diagram of vorticity for hydrodynamics. (f) Space time diagram of vorticity for MHD. The perturbation wavenumber is $m=$ 256 and the plasma beta is $\beta=4$ for MHD. . . . . . . . . . . . . . . 55

4.14 Time history of circulation in the whole domain for hydrodynamics and MHD. The perturbation wavenumber is $m=256$ and the plasma beta is $\beta=4$ for MHD. . . . . . . . . . . . . . . . . . . 56 


\section{LIST OF TABLES}

3.1 Numeric convergence test table (Hydrodynamics). The test was performed at time $t=0.12$. . . . . . . . . . . . . . . . . . . . . 35

3.2 Numeric convergence test table (MHD). The test was performed at time $t=0.12 \ldots \ldots \ldots \ldots$. . . . . . . . . . . . 36

$4.1 \quad$ Parameters of investigation for a single shocked interface case. . . . . 43

4.2 Exponential curve fit results of RTI for different wavenumber $m$. . . 47

A.1 Comparison between Algorithm 1 and Chisnell's Paper. . . . . . . . . 66 


\section{Chapter 1}

\section{Introduction}

The Richtmyer-Meshkov instability (RMI) is of great importance in experimental, theoretical and computational research because it is related to a wide variety of subjects such as inertial confinement fusion (ICF) [1] and astrophysical phenomena[2]. The RMI occurs when an incident shock crosses a corrugated interface between two fluids of different densities. Richtmyer [3] originally developed the linear theory of RMI. Then Meshkov[4] confirmed this prediction experimentally. Richtmyer proposed the impulsive model and concluded that the perturbation grew linearly with time at the contact discontinuity. Richtmyer's theory was developed in the case of a reflected shock. The linear analysis of RMI when the reflected wave is a rarefaction wave was developed by Yang et al.[5].

Applying magnetic field effects on the RMI was first introduced by Samtaney[6]. He showed by numerical simulations that the RMI could be suppressed in the presence of a magnetic field. This was further reconfirmed by analytical incompressible Magnetohydrodynamics (MHD) theory of an impulsively accelerated interface developed by Wheatley et al. [7, 8, 9]. In Samtaney's work, the magnetic field was aligned to the motion of the shock, the suppression of the RMI was caused by the bifurcation of the vortex sheet which transported the baroclinically generated vorticity away from the contact discontinuity by a pair of MHD shocks. Recently, the idea of using magnetic fields in RMI has suddenly become popular. Sano et al.[10] performed a 
numerical simulation and found that due to the fluid motions associated with the RMI, the ambient magnetic field strength was amplified substantially by an order of two magnitude. Hohenberger et al. [11] concluded experimentally that compared to the unmagnetized explosion in ICF, the ion temperature was increased by $15 \%$ in the presence of magnetic field, which improved the ICF performance.

A lot of theoretical and numerical studies have been performed in plane geometry. However, in ICF, the RMI occurs in a curved geometry. The first task of studying RMI in a curved geometry is to generate a clean shock. Chisnell[12] gave an analytic description of converging shock in cylindrical and spherical geometry. But his theory was only valid for strong shocks. Ponchaut et al. 13] adopted the method of series expansion and developed a numerical, characteristics based solution which was also valid for weak shocks. In the context of the RMI, Lombardini and Pullin[14] developed the theory for asymptotic growth rate in density interface for a three dimensional cylindrical geometry. In a converging geometry, not only do we have the RMI due to initial shock impact, but also the acceleration of the interface leads to an Rayleigh-Taylor instability (RTI) phase which changes the growth rate of the interface perturbations.

To the best of our knowledge, the RMI study in cylindrical geometry with the effects of MHD has not been performed. In this thesis, we examine the linear stability of an azimuthally perturbed density interface accelerated by a shock wave in cylindrical geometry in both hydrodynamics and MHD. Our approach extends the numerical linear stability analysis of Samtaney[15] to MHD in cylindrical coordinates. We linearize the ideal MHD equations about a time dependent base state and derive a set of hyperbolic equations with source terms for the perturbed quantities. The equations are then solved using an upwind technique. We limit the scope of this thesis to linear simulations of interfaces with positive Atwood number and purely azimuthal perturbations interacting with Chisnell-type[12] shocks of fixed Mach number (see 
Chapter 4 for detailed description of parameters).

The physical setup of our problem is depicted in Fig. 1.1. A converging domain is confined by left boundary of $r=R_{l}$ and right boundary of $r=R_{r}$. A Chisnell-type converging shock of Mach number $M_{s}$ is generated at $r=R_{s}$, traversing inwards. A density interface of azimuthal perturbation wavenumber $m$ is located at $r=R_{0}$, separating two fluids of density $\rho_{1}$ and $\rho_{2}$. The magnetic field, if present, is along the radial direction and with an initial strength characterized by plasma beta $\beta$ (defined as $\beta=2 p / B_{r}^{2}$, where $p$ is the initial pressure and $B_{r}$ is the magnetic field strength in radial direction). Therefore, our problem can be characterized by five parameters. The Mach number $M_{s}$ parameterizes the shock speed. The Atwood number $A$ (defined as $\left.\left(\rho_{2}-\rho_{1}\right) /\left(\rho_{2}+\rho_{1}\right)\right)$ characterizes the contact density discontinuity. The perturbation wavenumber $m$ expresses the wavelength of perturbation. The plasma beta $\beta$ determines the magnetic field strength. Finally, the distance ratio $R_{s} / R_{0}$ describes the distance between shock and interface. The five parameters $\left(M_{s}, A, m, \beta, R_{s} / R_{0}\right)$ completely determine our solutions. In this work, we will mainly study the cases of different perturbation wavenumber $m$ and plasma beta $\beta$.

The rest of the thesis is organized as follows. In Chapter 2, we introduce the ideal MHD equations and then linearize them to a form suitable for numerical linear stability analysis. In Chapter 3, we briefly present the numerical methods adopted to solve the linearized MHD system. In Chapter 4, numerical results are presented and discussed. Finally, the last Chapter 5 is reserved for conclusions and future work. 


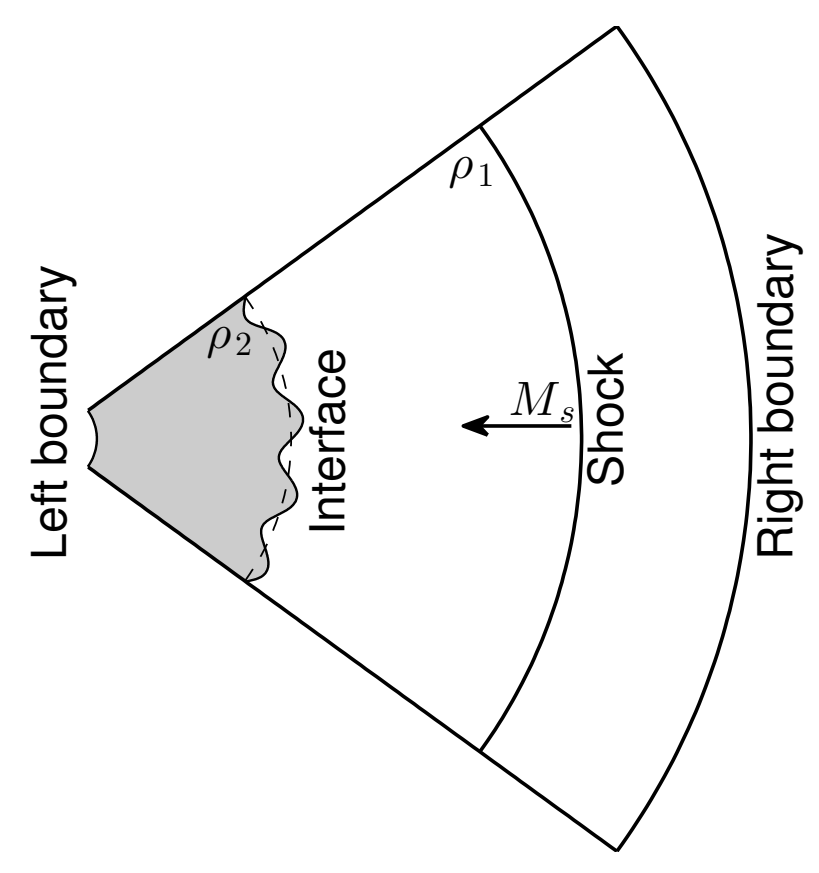

Figure 1.1: Illustration of the physical setup. A perturbed interface is located at $r=R_{0}$, separating two fluids of density $\rho_{1}$ and $\rho_{2}$. An incident shock is located at $r=R_{s}$, with Mach number $M_{s}$. The whole domain is bounded by left boundary and right boundary. 


\section{Chapter 2}

\section{Governing Equations}

\subsection{The Ideal MHD Equations}

The non-dimensional ideal MHD equations in conservative form in cylindrical coordinates are

$$
\frac{\partial U}{\partial t}+\frac{1}{r} \frac{\partial}{\partial r}(r F(U))+\frac{1}{r} \frac{\partial G(U)}{\partial \theta}=S
$$

where $r, \theta$ and $z$ are the cylindrical coordinates, and $t$ denotes time. $U \equiv U(r, \theta, t)$ is the solution vector and is written as

$$
U=\left\{\rho, \rho u_{r}, \rho u_{\theta}, \rho u_{z}, B_{r}, B_{\theta}, B_{z}, e\right\}^{T},
$$

where $\rho$ is the density, $\left(\rho u_{r}, \rho u_{\theta}, \rho u_{z}\right)$ is the momentum in the $r, \theta$ and $z$ directions, respectively. Similarly, $\left(B_{r}, B_{\theta}, B_{z}\right)$ is the magnetic field in the $r, \theta$ and $z$ directions. The $z$ component is retained because $u_{z}$ and $B_{z}$ can be nonzero in some cases. $e$ is the total energy per unit volume, including the magnetic energy term $\frac{1}{2}(\mathbf{B} \cdot \mathbf{B}) . F(U)$ 
and $G(U)$ are the fluxes in the $r$ and $\theta$ directions given by

$$
F=\left[\begin{array}{c}
\rho u_{r} \\
\rho u_{r}^{2}+\tilde{p}-B_{r}^{2} \\
\rho u_{r} u_{\theta}-B_{r} B_{\theta} \\
\rho u_{r} u_{z}-B_{r} B_{z} \\
0 \\
u_{r} B_{\theta}-u_{\theta} B_{r} \\
u_{r} B_{z}-u_{z} B_{r} \\
(e+\tilde{p}) u_{r}-(\mathbf{B} \cdot \mathbf{u}) B_{r}
\end{array}\right], G=\left[\begin{array}{c}
\rho u_{\theta} \\
\rho u_{r} u_{\theta}-B_{r} B_{\theta} \\
\rho u_{\theta}^{2}+\tilde{p}-B_{\theta}^{2} \\
\rho u_{\theta} u_{z}-B_{\theta} B_{z} \\
u_{\theta} B_{r}-u_{r} B_{\theta} \\
0 \\
u_{\theta} B_{z}-u_{z} B_{\theta} \\
(e+\tilde{p}) u_{\theta}-(\mathbf{B} \cdot \mathbf{u}) B_{\theta}
\end{array}\right]
$$

where $\mathbf{B} \cdot \mathbf{u}$ is the usual dot product defined as

$$
\mathbf{B} \cdot \mathbf{u}=B_{r} u_{r}+B_{\theta} u_{\theta}+B_{z} u_{z}
$$

$\tilde{p}$, the total pressure, is the sum of gas pressure and the magnetic pressure, i.e.,

$$
\tilde{p}=p+\frac{1}{2}(\mathbf{B} \cdot \mathbf{B})
$$

where $p$ is the gas pressure.

$S$ is the additional source term appearing in cylindrical coordinates. It is given 
by

$$
S=-\frac{1}{r}\left[\begin{array}{c}
0 \\
B_{\theta}^{2}-\rho u_{\theta}^{2}-\tilde{p} \\
\rho u_{r} u_{\theta}-B_{r} B_{\theta} \\
0 \\
0 \\
u_{\theta} B_{r}-u_{r} B_{\theta} \\
0 \\
0
\end{array}\right]
$$

To close the system, the equation of state is

$$
e=\frac{p}{\gamma-1}+\frac{1}{2}(\mathbf{B} \cdot \mathbf{B})+\frac{1}{2} \rho(\mathbf{u} \cdot \mathbf{u})
$$

where $\gamma$ is the ratio of specific heats. In what follows, we will always take $\gamma=5 / 3$ unless otherwise stated.

Gauss's law for magnetism states that the magnetic field has divergence equal to zero. This adds another constraint to the system

$$
\nabla \cdot \mathbf{B}=0
$$

This equation implies the magnetic field is in fact constrained. We will discuss more about this in Chapter 3 on numerical method.

\subsection{Linearization of the Ideal MHD Equations}

We linearize Eqn. (2.1) by splitting $U(r, \theta, t)=\stackrel{\circ}{U}(r, t)+\epsilon \hat{U}(r, t) e^{j m \theta}$, where the overcircle means base state and the over-hat means perturbed state. We shall adopt these notations throughout this work. The variable $m$ is perturbation wavenumber and $j$ is 
the imaginary number $\sqrt{-1}$. We split every term into a base and a perturbed state. Finally we get the following systems of equations, governing the base and perturbed solution.

$$
\begin{aligned}
& \frac{\partial \stackrel{\circ}{\partial}}{\partial t}+\frac{1}{r} \frac{\partial}{\partial r}(r F(\stackrel{\circ}{U}))=\stackrel{\circ}{S}, \\
& \frac{\partial \hat{U}}{\partial t}+\frac{1}{r} \frac{\partial}{\partial r}(r A(\stackrel{\circ}{U}) \hat{U})+\frac{1}{r} M(\stackrel{\circ}{U}) \hat{U} j m=\hat{S} .
\end{aligned}
$$

where $A\left(\dot{U}^{\circ}\right)$ is the Jacobian matrix of flux $F\left(\dot{U}^{\circ}\right)$ with respect to $\stackrel{\circ}{U}^{\text {, and }} M\left(\dot{U}^{\circ}\right)$ is the Jacobian matrix of flux $G(\stackrel{\circ}{U})$ with respect to $\stackrel{\circ}{U}$.

The two solution vectors are $\stackrel{\circ}{U}^{\text {and }} \hat{U}$. To derive them, take the second component

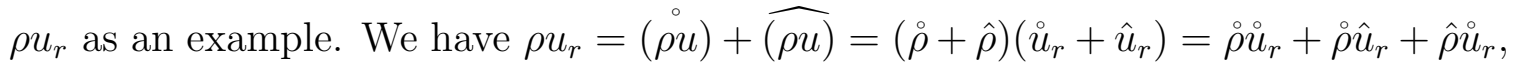
where we ignore the high order term $\hat{\rho} \hat{u}_{r}$. Therefore, $(\rho \dot{\circ})=\stackrel{\circ}{\rho} \stackrel{\circ}{r}_{r}$ and $\widehat{(\rho u)}=\stackrel{\circ}{\rho} \hat{u}_{r}+\hat{\rho} \stackrel{\circ}{u}_{r}$. The component expressions of solution vectors are written in Eqn. 2.10 .

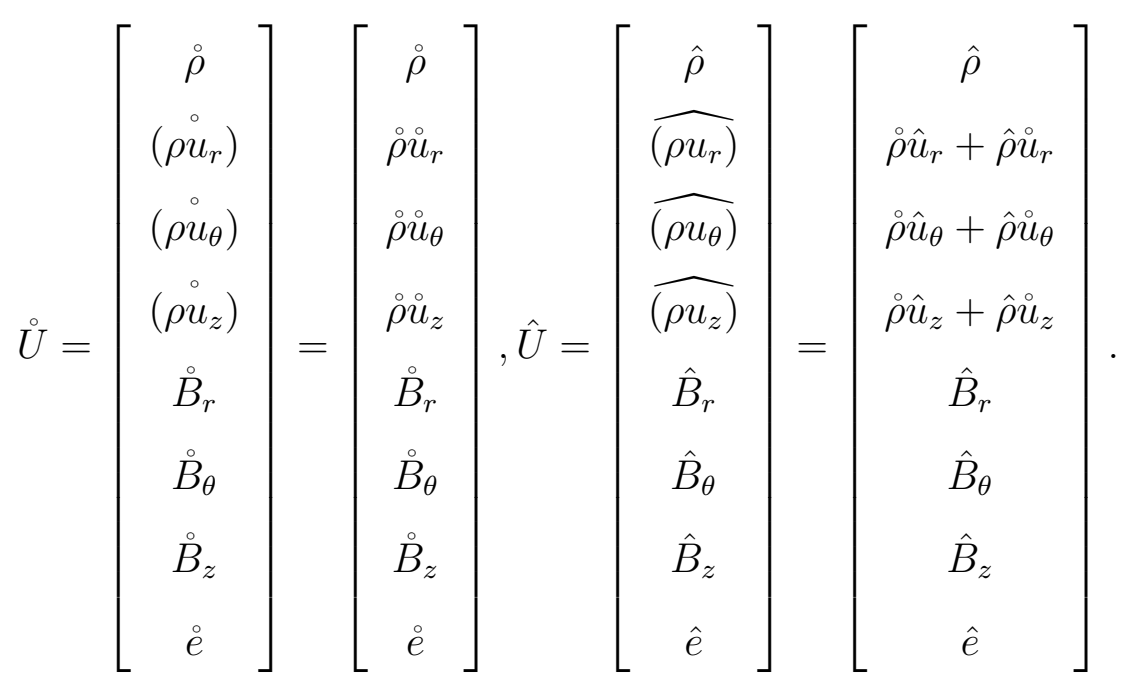


The component expressions of source terms are given in Eqn. 2.11

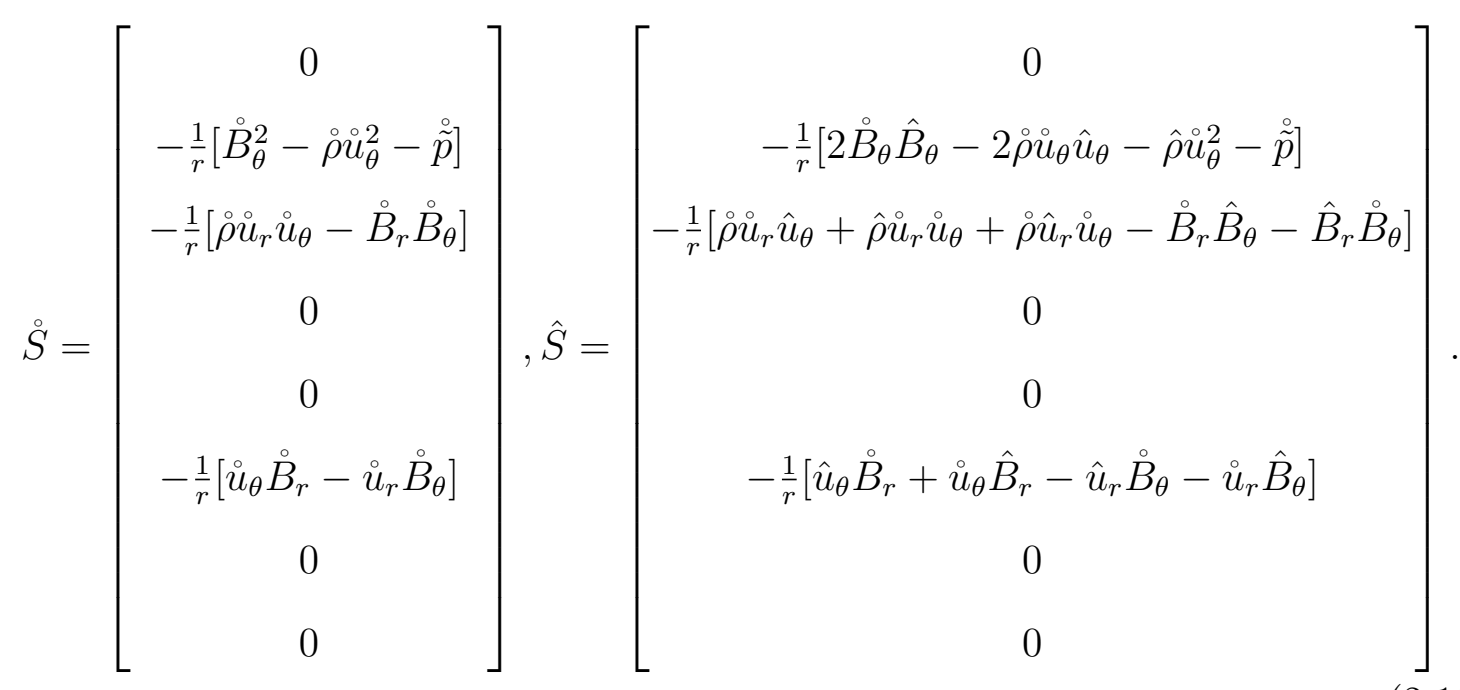

In the end, we need to specify the total pressure $\stackrel{\tilde{p}}{\text { and }} \hat{\tilde{p}}$ in term of other variables. These are written as:

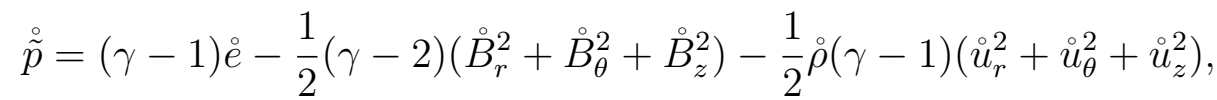

$$
\begin{aligned}
& \hat{\tilde{p}}=(\gamma-1) \hat{e}-(\gamma-2)\left(\stackrel{\circ}{B}_{r} \hat{B}_{r}+\stackrel{\circ}{B}_{\theta} \hat{B}_{\theta}+\stackrel{\circ}{B}_{z} \hat{B}_{z}\right)-\frac{1}{2} \hat{\rho}(\gamma-1)\left(\stackrel{\circ}{r}_{r}^{2}+\stackrel{\circ}{u}_{\theta}^{2}+\stackrel{\circ}{u}_{z}^{2}\right) \\
& -\stackrel{\circ}{\rho}(\gamma-1)\left(\stackrel{\circ}{u}_{r} \hat{u}_{r}+\stackrel{\circ}{u}_{\theta} \hat{u}_{\theta}+\stackrel{\circ}{u}_{z} \hat{u}_{z}\right) .
\end{aligned}
$$

\subsection{Scale free property of the Ideal MHD Equa- tions}

In this section, we show that the ideal MHD equations are scale free, i.e., after rescaling both time and space by a factor of $\alpha$, the solution remains unchanged. The proof is trivial. We will only consider the base state equation. The perturbed state equation is treated similarly. The base state equation is

$$
\frac{\partial \stackrel{\circ}{U}}{\partial t}+\frac{1}{r} \frac{\partial}{\partial r}(r F(\stackrel{\circ}{U}))=\stackrel{\circ}{S}
$$


We introduce the notation of prime. A variable will add a prime after it is rescaled. Let $t^{\prime}=\alpha t$ and $r^{\prime}=\alpha r$ where $\alpha$ is the scaling factor. Substitute into Eqn. 2.14

$$
\frac{\partial \stackrel{\circ}{U}^{\prime}}{\partial\left(\alpha t^{\prime}\right)}+\frac{1}{\alpha r^{\prime}} \frac{\partial}{\partial \alpha r^{\prime}}\left(\alpha r^{\prime} F\left(\stackrel{\circ}{U^{\prime}}\right)\right)=\stackrel{\circ}{S}
$$

Recall from Eqn. (2.11), there is a $1 / r$ factor in $\stackrel{\circ}{S}$ term. Finally, canceling $\alpha$ on both sides, we get

$$
\frac{\partial \dot{U}^{\prime}}{\partial t^{\prime}}+\frac{1}{r^{\prime}} \frac{\partial}{\partial r^{\prime}}\left(r^{\prime} F\left(\stackrel{\circ}{U}^{\prime}\right)\right)=\stackrel{\circ}{S}^{\prime}
$$

Eqn. 2.16 has the same expression as Eqn. 2.14). Therefore, we conclude that $\stackrel{\circ}{U}(r, \theta, t)=\stackrel{\circ}{U}^{\prime}\left(\alpha r^{\prime}, \theta, \alpha t^{\prime}\right)$. Because of this scale free property of ideal MHD equations, we can now fix our computational domain in the radial direction (such that the density interface is always at unit radius) and focus on the physical results. 


\section{Chapter 3}

\section{Numerical Method}

In this chapter, we describe the numerical method to solve the ideal MHD Eqn. (2.9).

The method we are using follows the approach of Samtaney[15].

\subsection{Temporal Discretization}

We use a "method of lines" approach and rewrite Eqn. 2.9a as

$$
\frac{\partial \stackrel{\circ}{\partial t}}{\partial t} R(\stackrel{\circ}{U})
$$

Assuming $\dot{U}^{n}$ is the solution at time step $n$ and $\dot{U}^{n+1}$ is the solution at a new time step $n+1$. We update the new solution $\dot{U}^{n+1}$ from $\dot{U}^{n}$ by the third order total variation diminishing (TVD) Runge-kutta time discretization approach.

$$
\begin{aligned}
& \stackrel{\circ}{U}_{0}=\stackrel{\circ}{U}^{n}, \\
& \stackrel{\circ}{U}_{1}=\stackrel{\circ}{U}_{0}+\Delta t R\left(\stackrel{\circ}{U}_{0}\right), \\
& \stackrel{\circ}{U}_{2}=\frac{3}{4} \stackrel{\circ}{U}_{0}+\frac{1}{4} \stackrel{\circ}{U}_{1}+\frac{\Delta t}{4} R\left(\stackrel{\circ}{U}_{1}\right), \\
& \stackrel{\circ}{U}_{3}=\frac{1}{3} \stackrel{\circ}{U}_{0}+\frac{2}{3} \stackrel{\circ}{U}_{2}+\frac{2 \Delta t}{3} R\left(\stackrel{\circ}{U}_{2}\right), \\
& \stackrel{\circ}{U}^{n+1}=\stackrel{\circ}{U}_{3} .
\end{aligned}
$$


where $\Delta t$ is the time step.

The perturbed state equation $(2.9 \mathrm{~b})$ is updated similarly.

\subsection{Spatial Discretization}

We adopt the finite volume method for spatial discretization. The computing domain $\left[R_{l}, R_{r}\right]$ is divided into $\mathrm{n}$ sub-intervals with equal length $\Delta r$. The centroid of each

interval is indexed as $i$ and the two neighboring faces are indexed as $i-\frac{1}{2}$ and $i+\frac{1}{2}$. The solution vectors $\stackrel{\circ}{U}$ and $\hat{U}$ are only defined at the centroids. The fluxes are computed at the faces and then used to update the solution at the centroids. An illustration is plotted in Fig. 3.1.

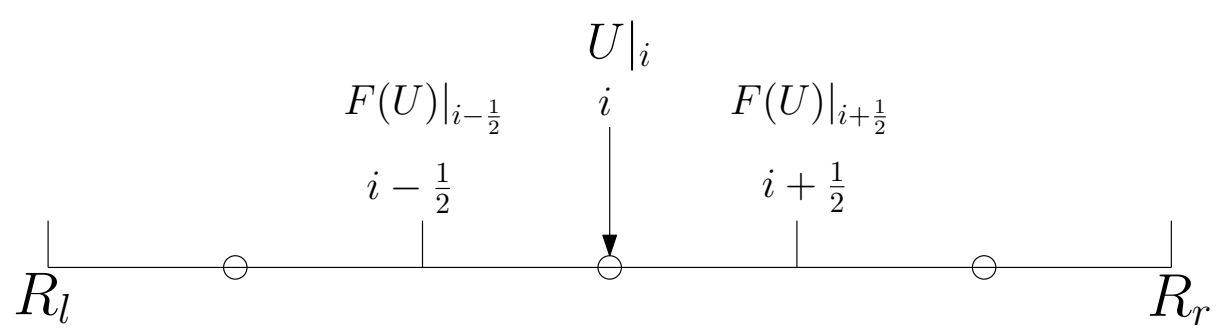

Figure 3.1: Illustration of spatial discretization. Circles are the centroids of each subintervals. The solution vectors are only defined at centroids. Fluxes are computed on two neighboring edges.

\subsection{Additional Stabilization Term}

We add the stabilization term $S_{\nabla \cdot \mathbf{B}}$ to the right hand side (RHS) of our system 2.1

$$
S_{\nabla \cdot \mathbf{B}}=-(\nabla \cdot \mathbf{B})\left[0, B_{r}, B_{\theta}, B_{z}, u_{r}, u_{\theta}, u_{z}, \mathbf{B} \cdot \mathbf{u}\right]^{T}
$$

The term $S_{\nabla \cdot \mathbf{B}}$ was originally introduced by Godunov[16] which results in an extra eigenvalue (equals $\stackrel{\leftrightarrow}{u}_{r}$ ) and is responsible for advecting $\nabla \cdot \mathbf{B}$ errors. We introduce this term following the approach of Powell et al.[17] and Falle et al.[18]. 
The governing equations are augmented with the above source term as

$$
\frac{\partial U}{\partial t}+\frac{1}{r} \frac{\partial}{\partial r}(r F(U))+\frac{1}{r} \frac{\partial G(U)}{\partial \theta}=S+S_{\nabla \cdot \mathbf{B}}
$$

We split it as we did before

$$
\begin{aligned}
& \frac{\partial \stackrel{\circ}{\partial t}}{\partial t}+\frac{1}{r} \frac{\partial}{\partial r}(r F(\stackrel{\circ}{U}))=\stackrel{\circ}{S}+\stackrel{\circ}{S}_{\nabla \cdot \mathbf{B}}, \\
& \frac{\partial \hat{U}}{\partial t}+\frac{1}{r} \frac{\partial}{\partial r}(r A(\stackrel{\circ}{U}) \hat{U})+\frac{1}{r} M(\stackrel{\circ}{U}) \hat{U} j m=\hat{S}+\hat{S}_{\nabla \cdot \mathbf{B}} .
\end{aligned}
$$

The two added stabilization source terms are given as

$$
\begin{aligned}
& \stackrel{\circ}{S}_{\nabla \cdot B}=\left[\begin{array}{c}
0 \\
-(\nabla \cdot \stackrel{\circ}{\mathbf{B}}) \stackrel{\circ}{B}_{r} \\
-(\nabla \cdot \stackrel{\circ}{\mathbf{B}}) \stackrel{\circ}{B}_{\theta} \\
-(\nabla \cdot \stackrel{\circ}{\mathbf{B}}) \stackrel{\circ}{B}_{z} \\
-(\nabla \cdot \stackrel{\circ}{\mathbf{B}}) \stackrel{\circ}{u}_{r} \\
-(\nabla \cdot \stackrel{\circ}{\mathbf{B}}) \stackrel{\circ}{u}_{\theta} \\
-(\nabla \cdot \stackrel{\circ}{\mathbf{B}}) \stackrel{\circ}{u}_{z} \\
-(\nabla \cdot \stackrel{\circ}{\mathbf{B}})[\stackrel{\circ}{\mathbf{B}} \cdot \stackrel{\circ}{\mathbf{u}}]
\end{array}\right]
\end{aligned}
$$

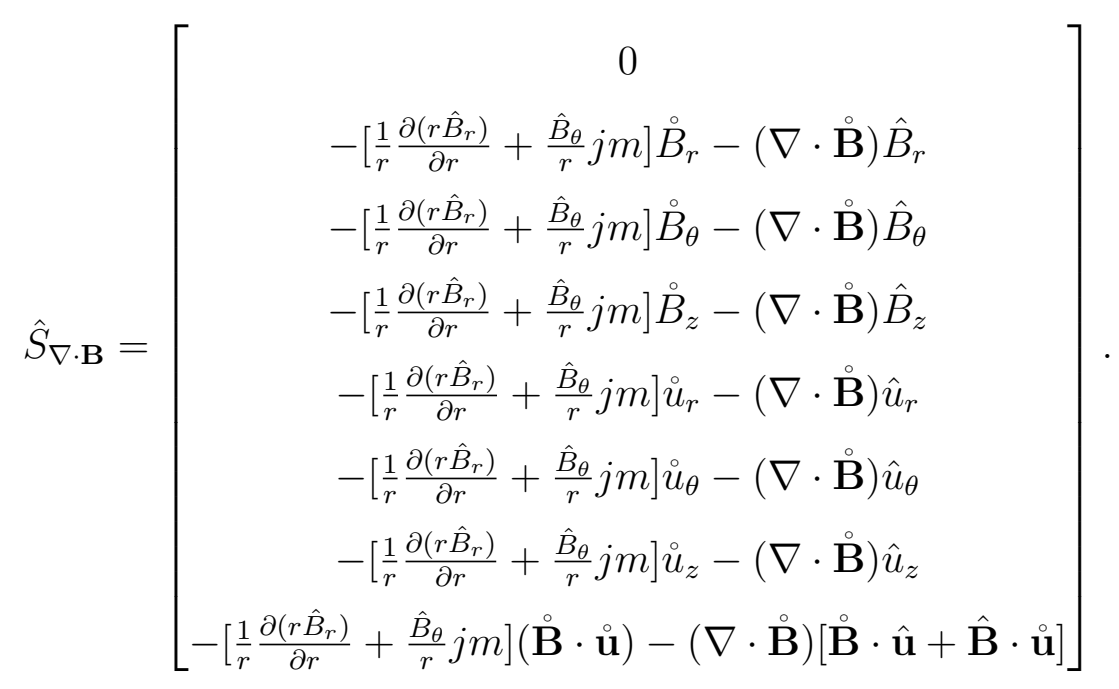




\subsection{Seven Wave Method}

The Jacobian matrix $A$ is singular. Therefore, to compute the fluxes, we use seven

wave method[19]. The solution vectors $\stackrel{\circ}{U}$ and $\hat{U}$ are rewritten to $\dot{U}$ and $\bar{U}$ to exclude the $B_{r}$ related row (the fifth row). Similarly, the Jacobian matrices $A\left(\dot{U}^{\circ}\right)$ and $M\left(\dot{U}^{\circ}\right)$ are rewritten to $\bar{A}\left({ }^{U}\right)$ and $\bar{M}\left(\stackrel{\circ}{U}^{\prime}\right)$ to exclude the $B_{r}$ related row and column (the fifth row and the fifth column).

For base state, the magnetic field is not time dependent for the chosen form of $\stackrel{\circ}{B}_{r}$ which is inversely proportional to radius. For perturbed state, after we exclude the $\hat{B}_{r}$ equation, the contribution of $\hat{B}_{r}$ to other variables still needs to be considered. Therefore, we add a correction term $C\left(\dot{U}, \hat{B}_{r}\right)$ to the RHS of perturbed state Eqn. (2.9b). The correction term is

$$
C\left(\stackrel{\circ}{U}, \hat{B}_{r}\right)=\left[0, \gamma \stackrel{\circ}{B}_{r}, \stackrel{\circ}{B}_{\theta}, \stackrel{\circ}{B}_{z}, \stackrel{\circ}{u}_{\theta}, \stackrel{\leftrightarrow}{u}_{z},\left(\gamma \stackrel{\circ}{B}_{r} \stackrel{\leftrightarrow}{u}_{r}+\stackrel{\circ}{B}_{\theta} \stackrel{\circ}{u}_{\theta}+\stackrel{\circ}{B}_{z} \stackrel{\circ}{u}_{z}\right)\right]^{T} \frac{1}{r} \frac{\partial\left(r \hat{B}_{r}\right)}{\partial r}
$$

This is similar to the approach adopted by Samtaney[15]. The perturbed state Eqn. 2.9b now become

$$
\frac{\partial \overline{\hat{U}}}{\partial t}+\frac{1}{r} \frac{\partial}{\partial r}(r \bar{A}(\stackrel{\circ}{U}) \overline{\hat{U}})+\frac{1}{r} \bar{M}(\stackrel{\circ}{U}) \overline{\hat{U}} j m=\overline{\hat{S}}+\overline{\hat{S}}_{\nabla \cdot \mathbf{B}}+C\left(\stackrel{\circ}{U}, \hat{B}_{r}\right)
$$

\subsubsection{Numerical flux}

In this section, we present methods to compute numerical fluxes in both the base state equation and perturbed state equation. The derivative of flux in the base state 
equation is numerically given by

$$
\begin{aligned}
\left.\frac{1}{r} \frac{\partial[r \bar{F}(\stackrel{\circ}{U})]}{\partial r}\right|_{i} & =\left.\frac{1}{r}\right|_{i} \frac{\left.[r \bar{F}(\stackrel{\circ}{U})]\right|_{i+\frac{1}{2}}-\left.[r \bar{F}(\stackrel{\circ}{U})]\right|_{i-\frac{1}{2}}}{\Delta r} \\
& =\left.\frac{1}{r}\right|_{i} \frac{\left.\left.r\right|_{i+\frac{1}{2}} \bar{F}(\stackrel{\circ}{U})\right|_{i+\frac{1}{2}}-\left.\left.r\right|_{i-\frac{1}{2}} \bar{F}(\stackrel{\circ}{U})\right|_{i-\frac{1}{2}}}{\Delta r}
\end{aligned}
$$

The flux $\left.\bar{F}(\stackrel{\circ}{U})\right|_{i+\frac{1}{2}}$ is evaluated as

$$
\left.\bar{F}(\stackrel{\circ}{U})\right|_{i+\frac{1}{2}}=\frac{1}{2}\left(\bar{F}\left(\stackrel{\bar{\sigma}}{U}_{L, i+\frac{1}{2}}, \stackrel{\circ}{B}_{r}\right)-\bar{F}\left(\stackrel{\bar{U}}{R}_{R, i+\frac{1}{2}}, \stackrel{\circ}{B}_{r}\right)\right)-\frac{1}{2} \sum_{k=1}^{7} \alpha_{k} \mathbf{r}_{k}
$$

where $\mathbf{l}_{k}$ and $\mathbf{r}_{k}$ are the left and right eigenvectors of $\bar{A} . \alpha_{k}$ is given by

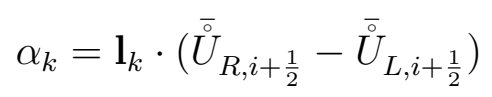

The $\stackrel{\bar{\ominus}}{R}_{R, i+\frac{1}{2}}$ and $\stackrel{\bar{o}}{U}_{L, i+\frac{1}{2}}$ are the left base state and right base state at the face $i+\frac{1}{2}$. They are obtained by fitting linear profiles and a Van-Leer slope limiter, as in Samtaney [15. The last term in Eqn. (3.11) is responsible for upwind and introduces dissipation in the codes. The upwind is useful for shock capture in the base state.

Similarly, the derivative of flux in the perturbed state equation is given by

$$
\begin{aligned}
\left.\frac{1}{r} \frac{\partial}{\partial r}[r \bar{A}(\stackrel{\circ}{U}) \overline{\hat{U}}]\right|_{i} & =\left.\frac{1}{r}\right|_{i} \frac{\left.[r \bar{A}(\stackrel{\circ}{U}) \overline{\hat{U}}]\right|_{i+\frac{1}{2}}-\left.[r \bar{A}(\stackrel{\circ}{U}) \overline{\hat{U}}]\right|_{i-\frac{1}{2}}}{\Delta r} \\
& =\left.\frac{1}{r}\right|_{i} \frac{\left.\left.r\right|_{i+\frac{1}{2}}[\bar{A}(\stackrel{\circ}{U}) \overline{\hat{U}}]\right|_{i+\frac{1}{2}}-\left.\left.r\right|_{i-\frac{1}{2}}[\bar{A}(\stackrel{\circ}{U}) \overline{\hat{U}}]\right|_{i-\frac{1}{2}}}{\Delta r}
\end{aligned}
$$


The flux term $\left.[\bar{A}(\stackrel{\circ}{U}) \overline{\hat{U}}]\right|_{i+\frac{1}{2}}$ is computed as

$$
\left.[\bar{A}(\stackrel{\circ}{U}) \overline{\hat{U}}]\right|_{i+\frac{1}{2}}=\frac{1}{2}\left(\bar{A}_{i+\frac{1}{2}} \overline{\hat{U}}_{L, i+\frac{1}{2}}+\bar{A}_{i+\frac{1}{2}} \overline{\hat{U}}_{R, i+\frac{1}{2}}\right)-\frac{1}{2}|\bar{A}|_{i+\frac{1}{2}}\left(\overline{\hat{U}}_{R, i+\frac{1}{2}}-\overline{\hat{U}}_{L, i+\frac{1}{2}}\right)
$$

Where $|\bar{A}|=\bar{A}^{+}-\bar{A}^{-}$. The $\overline{\hat{U}}_{R, i+\frac{1}{2}}$ and $\overline{\hat{U}}_{L, i+\frac{1}{2}}$ are left perturbed state and right perturbed state at the face $i+\frac{1}{2}$. These are obtained by fitting linear profiles and Van-Leer slope limiter. The slope fitting and limiting processes is done by first projecting the primitive variables to characteristic space by multiplying by the left eigenvectors of $\bar{A}$, computing the slopes in characteristic space, applying the limiter and then projecting back to physical space by multiplying by the right eigenvectors of $\bar{A}$. The last term in Eqn. (3.14) is the dissipative and upwind term, which is useful to reduce dissipative errors in the perturbed solution.

\subsubsection{Preserving the Solenoidal Property of the Magnetic Field}

Because of the divergence free constraint on magnetic field, the perturbed variable $\hat{B}_{\theta}$ has to be updated correctly. Recall that

$$
\nabla \cdot \mathbf{B}=0
$$

i.e.,

$$
\frac{1}{r} \frac{\partial}{\partial r}\left(r B_{r}\right)+\frac{1}{r} \frac{\partial B_{\theta}}{\partial \theta}=0
$$

Splitting into base state and perturbed state

$$
\frac{\partial}{\partial r}\left(r \stackrel{\circ}{B}_{r}\right)+\frac{1}{r} \frac{\partial \stackrel{\circ}{B}_{\theta}}{\partial \theta}=0, \quad \frac{\partial}{\partial r}\left(r \hat{B}_{r}\right)+j m \hat{B}_{\theta}=0 .
$$


The first equation shows magnetic field in the base state is divergence free. We will

choose $\stackrel{\circ}{B}_{\theta}=0$ throughout this thesis so that $\stackrel{\circ}{B}_{r}$ is inversely proportional to the radius. The second equation is used to update $\hat{B}_{\theta}$ by

$$
\left.\hat{B}_{\theta}\right|_{i}=\left.\frac{j}{m} \frac{\partial}{\partial r}\left(r \hat{B}_{r}\right)\right|_{i} \approx \frac{j}{m} \frac{\left.r \hat{B}_{r}\right|_{i+1}-\left.r \hat{B}_{r}\right|_{i-1}}{2 \Delta r}
$$

Both $\hat{B}_{r}$ and $\hat{B}_{\theta}$ are in the same time step. This procedure is performed at the end stage of each time step.

\subsection{Passive Scalar to Track Density Interface}

We are interested in the time history of perturbation growth rate at the interface. This requires that the location of the interface be computed at every time step. Therefore, we add another variable, a passive scalar $\psi$, to the system. The transportation equation governing passive scalar $\psi$ is

$$
\frac{\partial \psi}{\partial t}+\mathbf{u} \cdot \nabla \psi=0
$$

$\psi=\psi(r, t)$ is a function of radial position and time. We append the Eqn. (3.19) to the system (2.1). Initially, $\psi(r, 0)$ is given as $-1(1)$ on the left(right) side of the interface, respectively. Fig. 3.5 plots an example profile of $\psi$. The interface is located at $R_{0}=1.0$.

We define the location $r_{*}$, which satisfies $\psi\left(r_{*}, t\right)=0$, as the location of the interface at time $t$. Because the interface does not coincide with the centroid of a cell, second order interpolation is employed to find $r_{*}$ and then the location is used again to quantify the growth rate at the interface. 


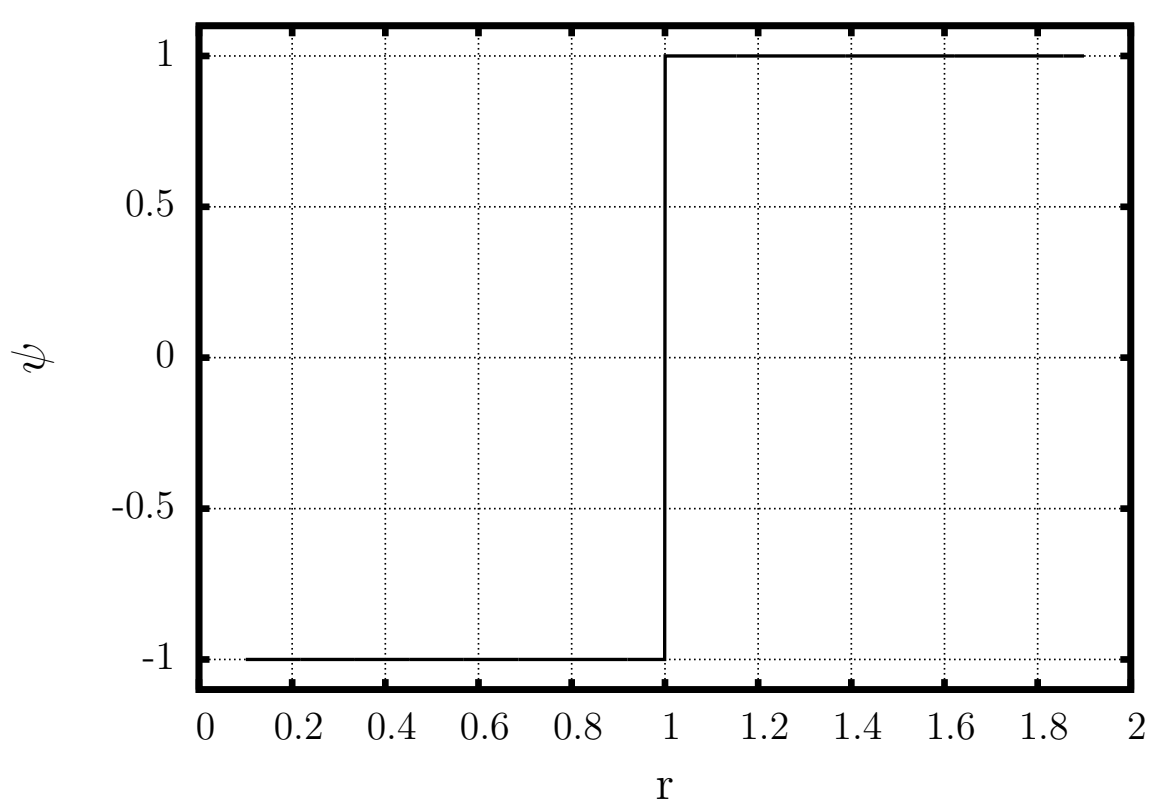

Figure 3.2: Illustration of passive scalar $\psi$ to track density interface. The interface is located at $R_{0}=1.0$.

\subsection{Convergence test}

In this section, two convergence tests are performed to quantify the order of accuracy of our numerical methods.

\subsubsection{Numerical convergence test}

In the numerical convergence test, we are interested in the order of accuracy of the perturbed state. Therefore, we set the base state to be constant over space and "steady" over time. The "steady" means that the base state is not updated during every time step. In our test, the computational domain is set to be $\left[R_{l}, R_{r}\right]=[3,7]$. The density $\stackrel{\circ}{\rho}$ and pressure $\stackrel{p}{p}$ are both set to 1.0 . The radial velocity $\stackrel{\circ}{r}_{r}$ is set to 0.3 . The magnetic field is set to zero for hydrodynamics. For MHD, $\beta$ is set to 16 at the interface position $R_{0}=5.0$. We choose the perturbation wavenumber $m=32$. The 


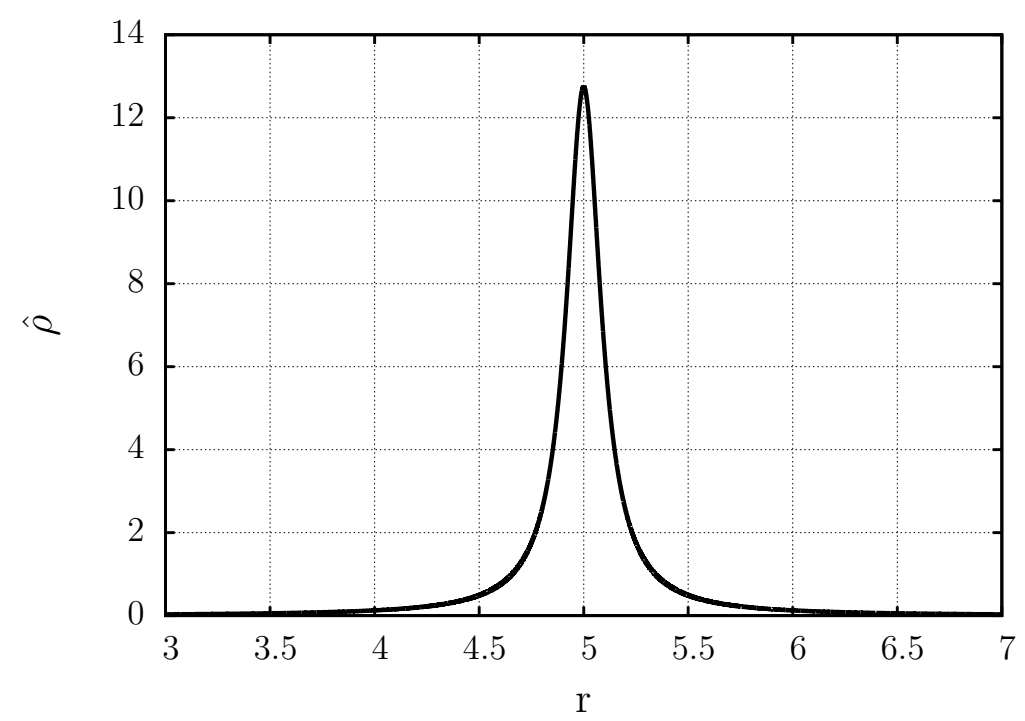

Figure 3.3: The initial profile of perturbed density $\hat{\rho}$ at time $t=0.00$ for numerical convergence test.

perturbed density is initialized by

$$
\hat{\rho}=\frac{\rho_{2}-\rho_{1}}{\pi \alpha\left\{1+\left(\frac{r-R_{0}}{\alpha}\right)^{2}\right\}}+j \frac{\rho_{2}-\rho_{1}}{\pi \alpha\left\{1+\left(\frac{r-R_{0}}{\alpha}\right)^{2}\right\}}
$$

We choose $\rho_{2}=5.0, \rho_{1}=1.0, R_{0}=5.0$ and $\alpha=0.1$ in order to get a smooth profile. Fig. 3.3 plots the initial profile for $\hat{\rho}$. Other conserved perturbed variables are set to zero.

The convergence tests for both hydrodynamics $(\beta=\infty)$ and $\operatorname{MHD}(\beta=16)$ are performed. For the hydrodynamics, the convergence order is around two. In Fig. 3.4 , we plot the profiles of perturbed variables $\hat{u}_{r}, \widehat{\rho u}_{r}, \widehat{\rho u}_{\theta}$ and $\hat{e}$ at time $t=0.12$ for grid sizes $400,800,1600,3200$ and 6400 . These figures show that the profiles of perturbed variables show virtually no difference between the coarsest mesh 400 and the finest mesh 6400. In Table 3.1, the convergence test is performed at time $t=0.12$ for mesh size 400, 800, 1600 and 3200. The numeric solution of mesh size 6400 was assumed to be the "exact" solution and is used to compute error norm for other mesh sizes. In 
the convergence table, we multiply every variable by a factor of radius $r$, to consider the contribution of convergent geometry. The $L_{1}$ and $L_{2}$ norms indicate approximate second order accuracy, as the mesh increases to 3200 and beyond.

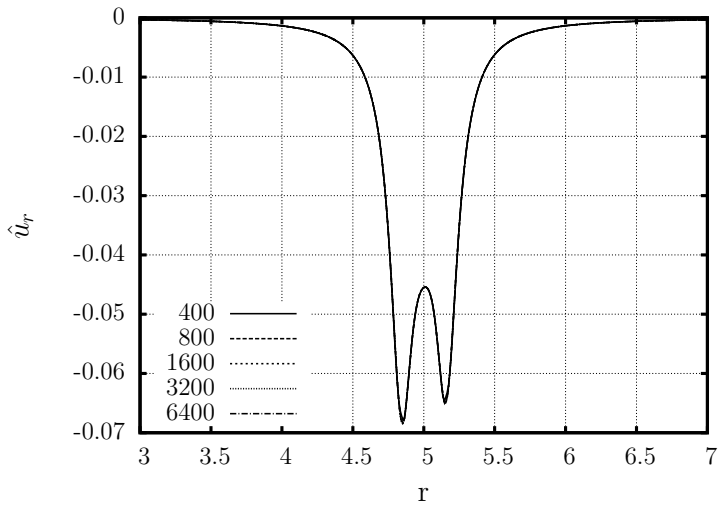

(a) Perturbed $r$-component of velocity $\hat{u}_{r}$

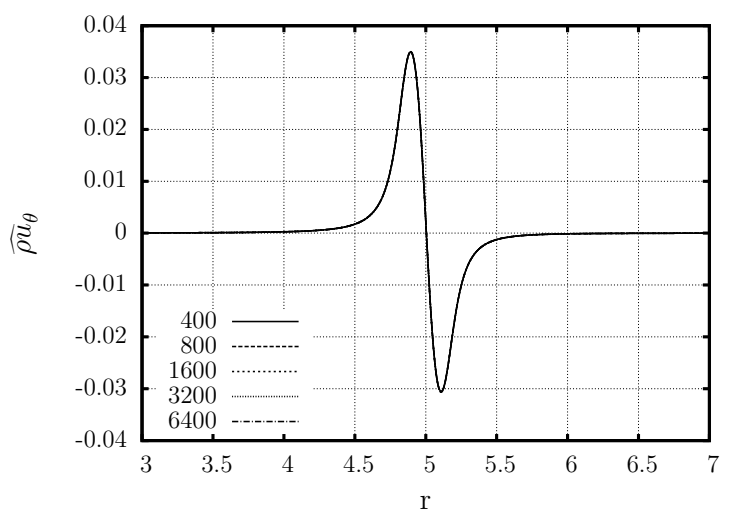

(c) Perturbed $\theta$-component of momentum $\widehat{\rho u}_{\theta}$

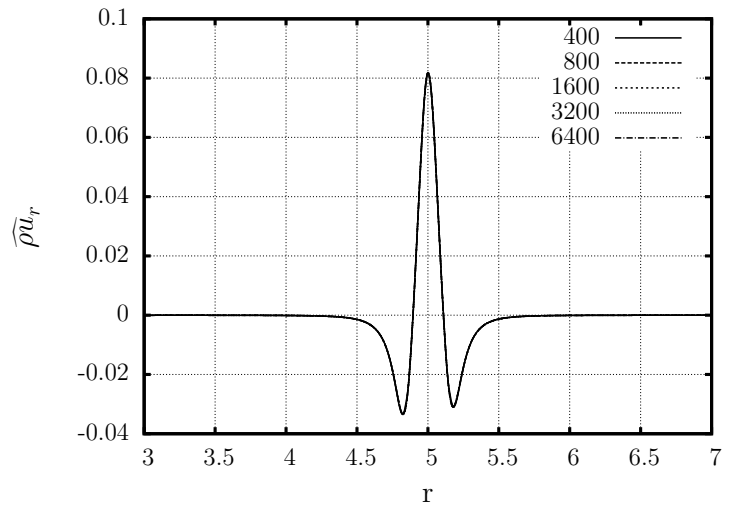

(b) Perturbed $r$-component of momentum $\widehat{\rho u}_{r}$

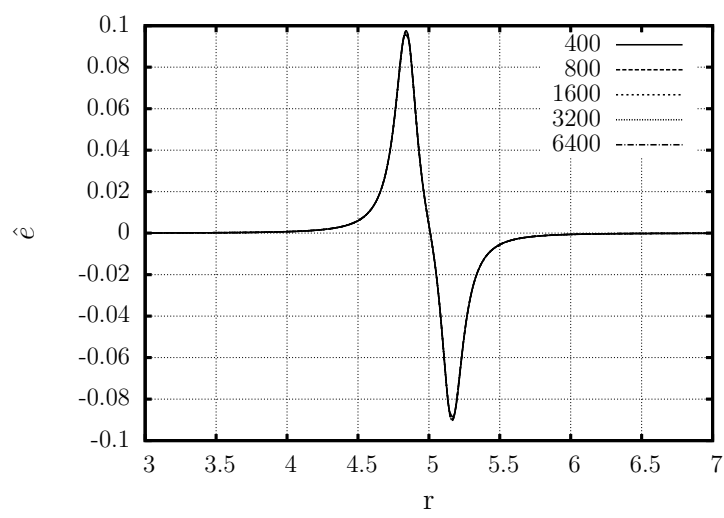

(d) Perturbed energy density $\hat{e}$

Figure 3.4: For hydrodynamics, the profiles of (a) perturbed $r$-component of velocity $\hat{u}_{r}$ (b) perturbed $r$-component of momentum $\hat{\rho} \hat{u}_{r}$ (c) perturbed $\theta$-component of momentum $\hat{\rho} u_{\theta}(\mathrm{d})$ perturbed energy density $\hat{e}$ at time $t=0.12$ for mesh size 400, 800, 1600,3200 and 6400 .

For the MHD, almost every variable listed has second order accuracy. $\hat{B}_{r}$ has convergence order of around 1.8. To show that the profiles of perturbed variables are almost identical for both the coarsest mesh 400 and the finest mesh 6400, we plot profiles of perturbed variables $\hat{u}_{r}, \widehat{\rho u}_{r}, \widehat{\rho u}_{\theta}, \hat{e}, \hat{B}_{r}$ and $\hat{B}_{\theta}$ at time $t=0.12$ for grid sizes 400, 800, 1600, 3200 and 6400 in Fig. 3.5. In Table 3.2, the convergence test is performed at time $t=0.12$ for mesh size 400, 800, 1600 and 3200. Some quantities 
Table 3.1: Numeric convergence test table (Hydrodynamics). The test was performed at time $t=0.12$

\begin{tabular}{|c|r|l|l|l|}
\hline Variable & Mesh & $L_{\infty}$ & $L_{1}$ & $L_{2}$ \\
\hline$r \hat{u}_{r}$ & 3200 & $1.77 \times 10^{-4}(1.82)$ & $1.22 \times 10^{-5}(2.04)$ & $2.48 \times 10^{-5}(2.10)$ \\
& 1600 & $6.26 \times 10^{-4}(1.48)$ & $5.05 \times 10^{-5}(1.79)$ & $1.06 \times 10^{-4}(1.74)$ \\
& 800 & $1.74 \times 10^{-3}(1.47)$ & $1.75 \times 10^{-4}(1.73)$ & $3.56 \times 10^{-4}(1.68)$ \\
& 400 & $4.81 \times 10^{-3}(-)$ & $5.78 \times 10^{-4}(-)$ & $1.14 \times 10^{-3}(-)$ \\
$r \widehat{\rho u}_{r}$ & 3200 & $1.77 \times 10^{-4}(1.82)$ & $1.26 \times 10^{-5}(2.06)$ & $2.49 \times 10^{-5}(2.10)$ \\
& 1600 & $6.26 \times 10^{-4}(1.47)$ & $5.23 \times 10^{-5}(1.80)$ & $1.07 \times 10^{-4}(1.74)$ \\
& 800 & $1.73 \times 10^{-3}(1.44)$ & $1.82 \times 10^{-4}(1.72)$ & $3.57 \times 10^{-4}(1.68)$ \\
& 400 & $4.69 \times 10^{-3}(-)$ & $5.98 \times 10^{-4}(-)$ & $1.15 \times 10^{-3}(-)$ \\
$r \widehat{\rho u}_{\theta}$ & 3200 & $1.06 \times 10^{-5}(2.05)$ & $2.34 \times 10^{-6}(1.79)$ & $3.39 \times 10^{-6}(1.94)$ \\
& 1600 & $4.40 \times 10^{-5}(1.86)$ & $8.05 \times 10^{-6}(1.66)$ & $1.31 \times 10^{-5}(1.76)$ \\
& 800 & $1.60 \times 10^{-4}(1.85)$ & $2.54 \times 10^{-5}(1.87)$ & $4.41 \times 10^{-5}(1.89)$ \\
& 400 & $5.75 \times 10^{-4}(-)$ & $9.33 \times 10^{-5}(-)$ & $1.64 \times 10^{-4}(-)$ \\
$r \hat{e}^{-4}$ & 3200 & $3.44 \times 10^{-4}(1.83)$ & $2.29 \times 10^{-5}(2.07)$ & $4.78 \times 10^{-5}(2.10)$ \\
& 1600 & $1.22 \times 10^{-3}(1.48)$ & $9.63 \times 10^{-5}(1.81)$ & $2.06 \times 10^{-4}(1.74)$ \\
& 800 & $3.41 \times 10^{-3}(1.46)$ & $3.37 \times 10^{-4}(1.74)$ & $6.92 \times 10^{-4}(1.69)$ \\
& 400 & $9.39 \times 10^{-3}(-)$ & $1.13 \times 10^{-3}(-)$ & $2.23 \times 10^{-3}(-)$ \\
\hline
\end{tabular}

(such as $r \widehat{\rho u}_{\theta}$ ) show a unusual convergence trend, which is not well understood at present. Nonetheless, the order of accuracy is still close to two. 
Table 3.2: Numeric convergence test table (MHD). The test was performed at time $t=0.12$

\begin{tabular}{|c|c|c|c|c|}
\hline Variable & Mesh & $L_{\infty}$ & $L_{1}$ & $L_{2}$ \\
\hline \multirow[t]{4}{*}{$r \hat{u}_{r}$} & 3200 & $1.76 \times 10^{-4}(1.82)$ & $1.22 \times 10^{-5}(2.05)$ & $2.48 \times 10^{-5}(2.10)$ \\
\hline & 1600 & $6.23 \times 10^{-4}(1.48)$ & $5.04 \times 10^{-5}(1.79)$ & $1.06 \times 10^{-4}(1.74)$ \\
\hline & 800 & $1.73 \times 10^{-3}(1.46)$ & $1.75 \times 10^{-4}(1.73)$ & $3.55 \times 10^{-4}(1.68)$ \\
\hline & 400 & $4.79 \times 10^{-3}(-)$ & $5.80 \times 10^{-4}(-)$ & $1.14 \times 10^{-3}(-)$ \\
\hline \multirow[t]{4}{*}{$r \widehat{\rho u}_{r}$} & 3200 & $1.77 \times 10^{-4}(1.82)$ & $1.26 \times 10^{-5}(2.06)$ & $2.49 \times 10^{-5}(2.10)$ \\
\hline & 1600 & $6.23 \times 10^{-4}(1.47)$ & $5.23 \times 10^{-5}(1.80)$ & $1.06 \times 10^{-4}(1.74)$ \\
\hline & 800 & $1.73 \times 10^{-3}(1.43)$ & $1.82 \times 10^{-4}(1.72)$ & $3.56 \times 10^{-4}(1.68)$ \\
\hline & 400 & $4.67 \times 10^{-3}(-)$ & $5.98 \times 10^{-4}(-)$ & $1.14 \times 10^{-3}(-)$ \\
\hline \multirow[t]{4}{*}{$r \widehat{\rho u}_{\theta}$} & 3200 & $1.07 \times 10^{-5}(2.07)$ & $2.32 \times 10^{-6}(1.76)$ & $3.37 \times 10^{-6}(1.95)$ \\
\hline & 1600 & $4.50 \times 10^{-5}(1.89)$ & $7.87 \times 10^{-6}(1.65)$ & $1.30 \times 10^{-5}(1.79)$ \\
\hline & 800 & $1.66 \times 10^{-4}(1.86)$ & $2.48 \times 10^{-5}(1.98)$ & $4.50 \times 10^{-5}(1.96)$ \\
\hline & 400 & $6.06 \times 10^{-4}(-)$ & $9.75 \times 10^{-5}(-)$ & $1.75 \times 10^{-4}(-)$ \\
\hline \multirow[t]{4}{*}{$r \hat{e}$} & 3200 & $3.43 \times 10^{-4}(1.83)$ & $2.28 \times 10^{-5}(2.07)$ & $4.78 \times 10^{-5}(2.10)$ \\
\hline & 1600 & $1.22 \times 10^{-3}(1.48)$ & $9.61 \times 10^{-5}(1.81)$ & $2.06 \times 10^{-4}(1.75)$ \\
\hline & 800 & $3.40 \times 10^{-3}(1.46)$ & $3.36 \times 10^{-4}(1.74)$ & $6.90 \times 10^{-4}(1.69)$ \\
\hline & 400 & $9.37 \times 10^{-3}(-)$ & $1.12 \times 10^{-3}(-)$ & $2.23 \times 10^{-3}(-)$ \\
\hline \multirow[t]{4}{*}{$r \hat{B}_{r}$} & 3200 & $8.20 \times 10^{-7}(1.91)$ & $2.11 \times 10^{-7}(1.70)$ & $3.11 \times 10^{-7}(1.80)$ \\
\hline & 1600 & $3.09 \times 10^{-6}(1.82)$ & $6.85 \times 10^{-7}(1.57)$ & $1.14 \times 10^{-6}(1.65)$ \\
\hline & 800 & $1.09 \times 10^{-5}(1.94)$ & $2.03 \times 10^{-6}(1.82)$ & $3.57 \times 10^{-6}(1.78)$ \\
\hline & 400 & $4.18 \times 10^{-5}(-)$ & $7.17 \times 10^{-6}(-)$ & $1.23 \times 10^{-5}(-)$ \\
\hline \multirow[t]{4}{*}{$r \hat{B}_{\theta}$} & 3200 & $4.45 \times 10^{-6}(2.31)$ & $1.08 \times 10^{-6}(2.19)$ & $1.67 \times 10^{-6}(2.24)$ \\
\hline & 1600 & $2.21 \times 10^{-5}(2.07)$ & $4.92 \times 10^{-6}(2.02)$ & $7.88 \times 10^{-6}(2.05)$ \\
\hline & 800 & $9.27 \times 10^{-5}(2.02)$ & $2.00 \times 10^{-5}(2.08)$ & $3.26 \times 10^{-5}(2.09)$ \\
\hline & 400 & $3.75 \times 10^{-4}(-)$ & $8.49 \times 10^{-5}(-)$ & $1.39 \times 10^{-4}(-)$ \\
\hline
\end{tabular}




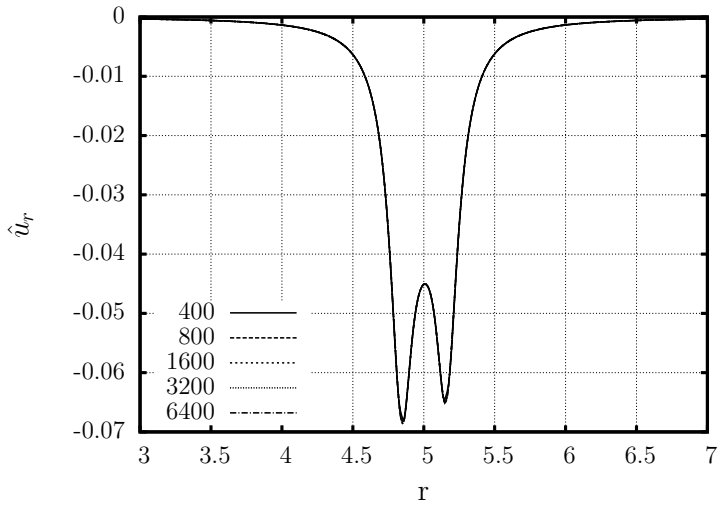

(a) Perturbed $r$-component of velocity $\hat{u}_{r}$

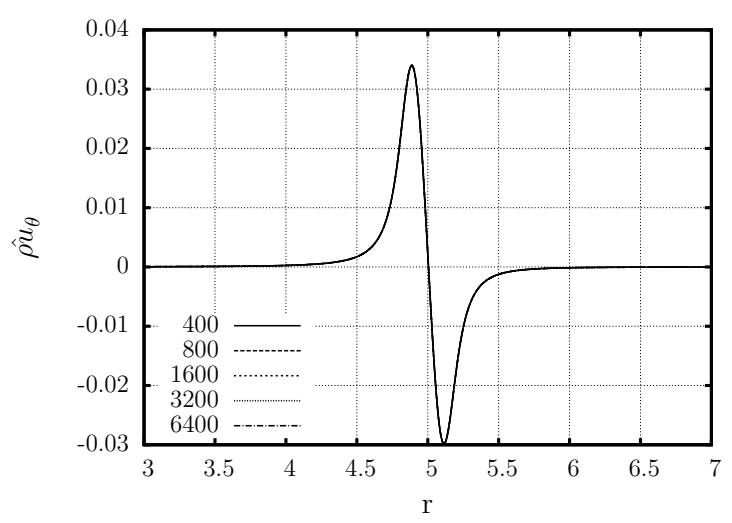

(c) Perturbed $\theta$-component of momentum $\widehat{\rho u}_{\theta}$

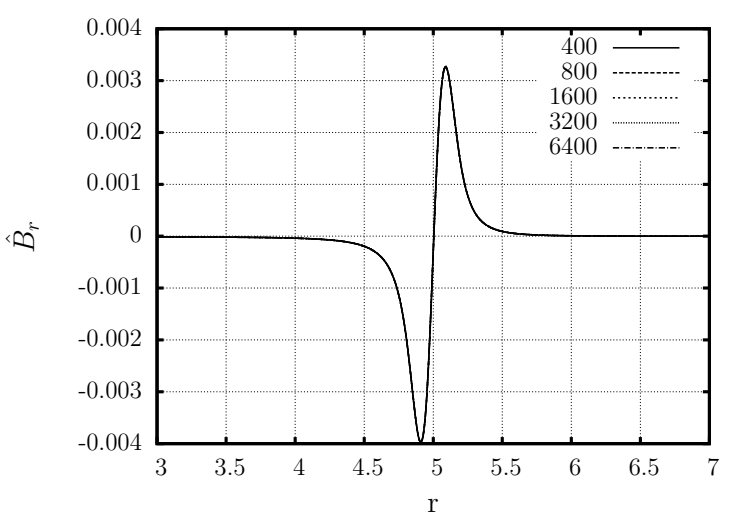

(e) Perturbed $r$-component of magnetic field $\hat{B}_{r}$

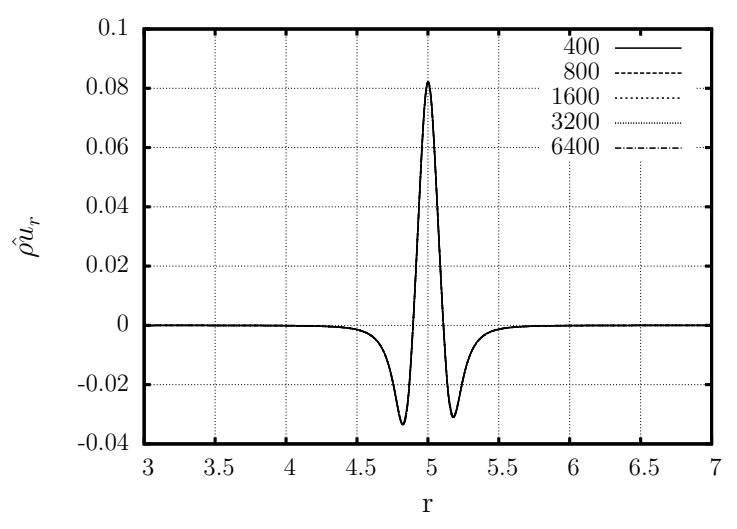

(b) Perturbed $r$-component of momentum $\widehat{\rho u}_{r}$

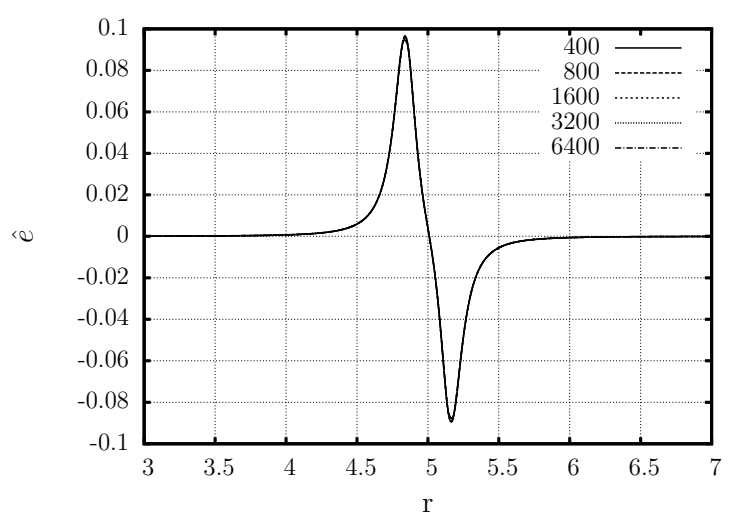

(d) Perturbed energy density $\hat{e}$

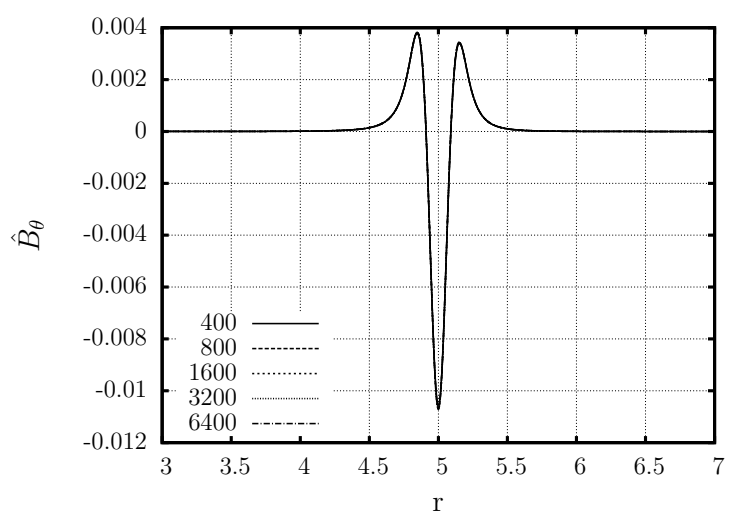

(f) Perturbed $\theta$-component of magnetic field $\hat{B}_{\theta}$

Figure 3.5: For MHD, the profiles of (a) perturbed $r$-component of velocity $\hat{u}_{r}$ (b) perturbed $r$-component of momentum $\hat{\rho} \hat{u}_{r}$ (c) perturbed $\theta$-component of momentum $\hat{\rho} u_{\theta}(\mathrm{d})$ perturbed energy density $\hat{e}(\mathrm{e})$ perturbed $r$-component of magnetic field $\hat{B}_{r}$ (f) perturbed $\theta$-component of magnetic field $\hat{B}_{\theta}$ at time $t=0.12$ for mesh size 400 , $800,1600,3200$ and 6400 . 


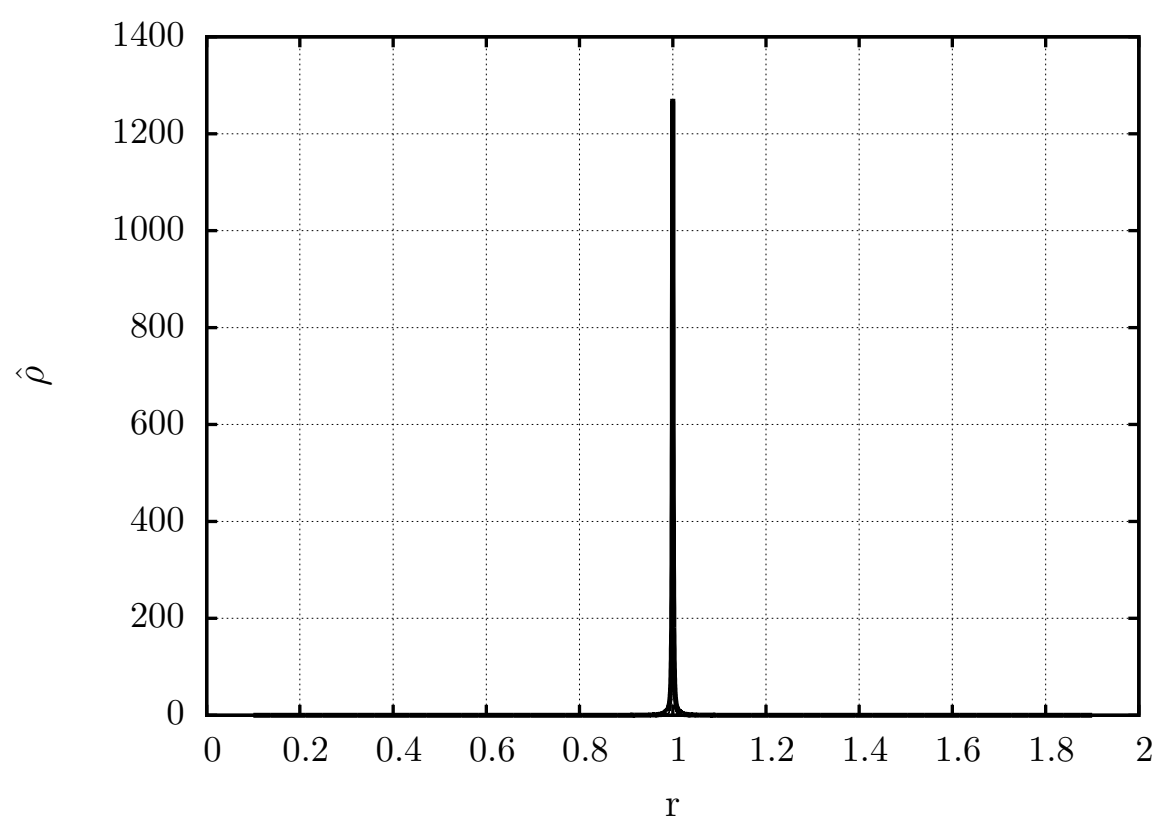

Figure 3.6: The initial profile of perturbed density $\hat{\rho}$ at time $t=0$ for a single shocked interface case. Profile is an approximation of a delta function

\subsubsection{Convergence test for a single shocked interface}

A convergence test is performed for a single-shocked interface case. The domain is set as $\left[R_{l}, R_{r}\right]=[0.1,1.9]$. A Chisnell-type shock of Mach 2.0 is initialized at $R_{s}=1.2$, moves radially to interface located at $R_{0}=1.0$ (Details of initialization of Chisnell shock are discussed in Appendix A). The azimuthal perturbation wavenumber is $m=32$. Eqn. 3.20 is used to generate the initial condition of $\hat{\rho} . \quad \rho_{1}$ and $\rho_{2}$ are chosen to be 1.0 and 5.0, respectively. $\alpha$ is selected to be 0.001 to obtain a sharp initial profile. The initial profile of $\hat{\rho}$ is plotted in Fig. 3.6 and approximately a delta function. This test is performed in hydrodynamics $(\beta=\infty)$.

When the shock traverses the interface, ideally the interface acquires an infinite large acceleration over zero time duration. But in our test, the acceleration is finite during a very short period of time. This is reflected in the growth rate plot 3.7 as a large spike shortly after the time that the shock hits the interface. This spike is of no real physical consequence. Fig. 3.7 shows the time history of unscaled perturbation 
growth rate $\dot{h}$ at interface for different mesh size 800, 1600, 3200, 6400, 12800. The growth rates virtually show no difference except for the coarsest mesh size 800 . A convergence test is performed at time $t=0.288$ for the perturbation growth rate $\dot{h}$. The numeric solution of mesh size 12800 is used as "exact" solution. The test results are showed in Fig. 3.8. The convergence order is somewhere between one and two, which is not surprising because of the presence of discontinuities.

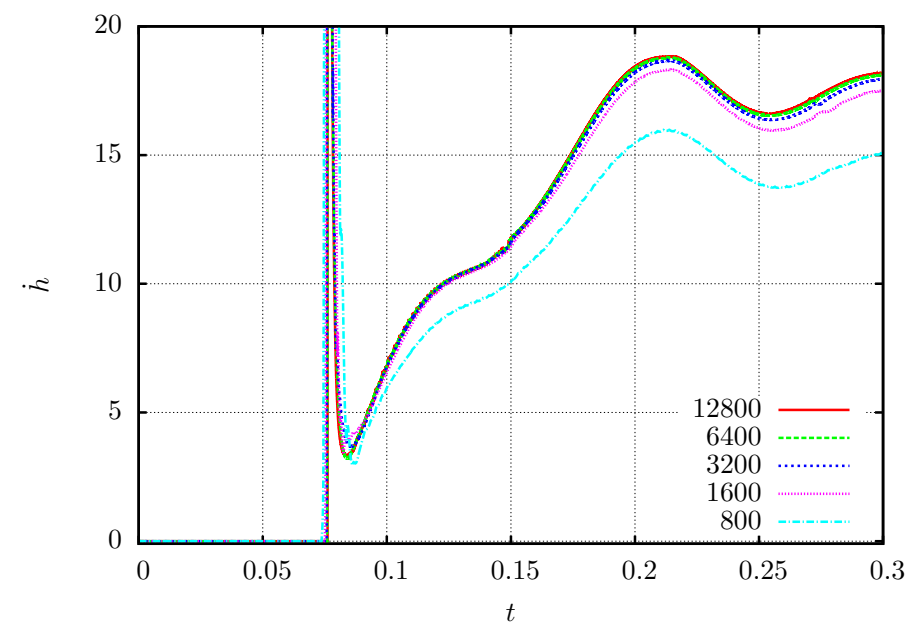

Figure 3.7: Time history of unscaled growth rate for the case of a single shocked interface using different mesh size 800, 1600, 3200, 6400, 12800.

To sum up, for smooth cases, the convergence rates are about two for $L_{1}$ and $L_{2}$ norms. For cases with discontinuities, the convergence rates for $L_{1}$ and $L_{2}$ norms are somewhere between one and two. 


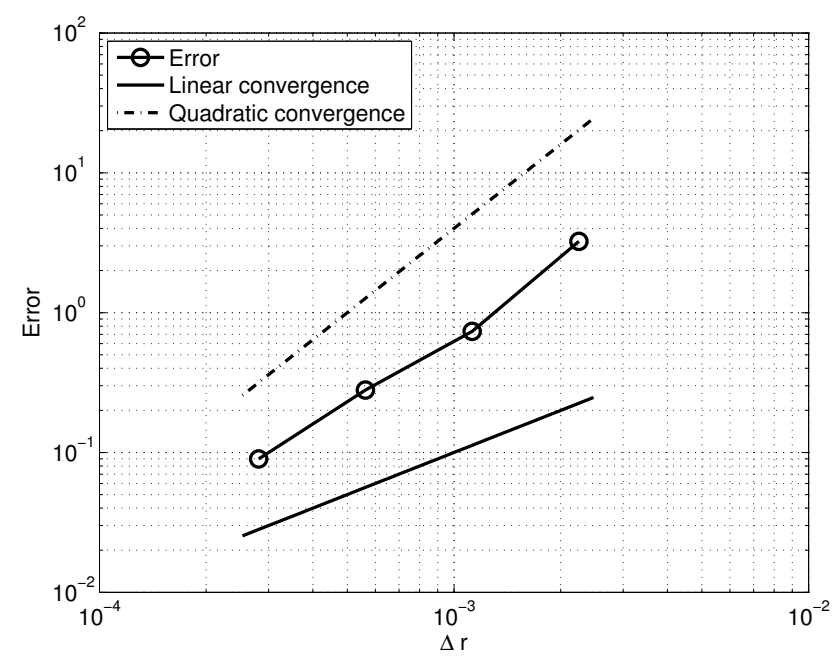

Figure 3.8: Convergence plot of unscaled growth rate for the case of a single shocked interface at time $t=0.288$. 


\section{Chapter 4}

\section{Linear Stability Results of RMI in Cylindrical Coordinates}

\subsection{Parameters of Investigation}

The physical setup we are interested is a Chisnell-type [12] shock, traversing from lighter density gas into heavier density gas, moving radially inwards to interact with the density interface (as in Fig 1.1). Here, we limit our investigation to purely azimuthal perturbations. We are interested in the perturbation growth rate history at the interface in the context of Richtmyer-Meshkov instability. The domain is $\left[R_{l}, R_{r}\right]=[0.1,1.9]$. A converging shock of Mach $M_{s}=2.0$ is initialized at $R_{s}=1.2$. The density of lighter (heavier) gas is set to be $1.0(5.0)$, respectively (Atwood number $A=0.667)$. The interface with perturbation wavenumber $m$ is located at $R_{0}=1.0$. For MHD, throughout this work, we will always assume magnetic field is only along radial direction and inversely proportional to the radius. The magnetic field strength is characterized by plasma beta $\beta=2 p / \stackrel{\circ}{r}_{r}^{2}$ at the interface. For the initial condition of perturbed state, the perturbed density $\hat{\rho}$ is

$$
\hat{\rho}=\frac{\rho_{2}-\rho_{1}}{\pi \alpha\left\{1+\left(\frac{R-R_{0}}{\alpha}\right)^{2}\right\}}+j \frac{\rho_{2}-\rho_{1}}{\pi \alpha\left\{1+\left(\frac{R-R_{0}}{\alpha}\right)^{2}\right\}}
$$


where $R_{0}=1.0$ is the interface position. $\rho_{2}=5.0\left(\rho_{1}=1.0\right)$ is the density on left (right) side of the interface. $\alpha$ is selected to be 0.001 so that initially the density perturbation approximates a delta function. The initial $\hat{\rho}$ profile is plotted in Fig. 4.1. Other conserved perturbed variables are set to zero. The boundary conditions are enforced through a ghost point technique. The solutions at ghost points are obtained by linear interpolation. In this thesis, we study the cases of different perturbation wavenumber and different magnetic field strength. The parameters of investigation are tabulated in table 4.1

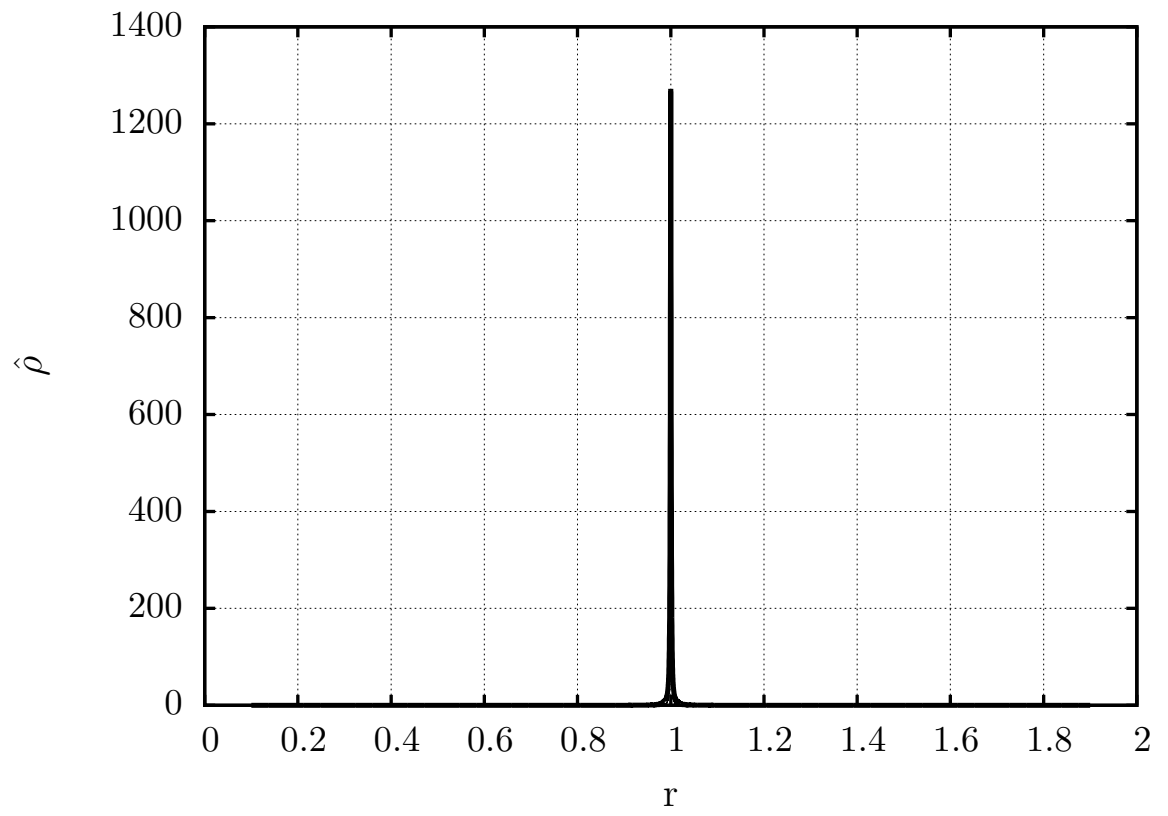

Figure 4.1: The initial profile of perturbed density $\hat{\rho}$ at time $t=0$ for a single shocked interface case. Profile is an approximation of a delta function

\subsection{Solution Details of Base State}

The solution of base state is independent of perturbation wavenumber $m$. This is because base state equations do not depend on perturbed state variables. Furthermore, for the chosen form of $\stackrel{\circ}{B}_{r}$ which is inversely proportional to radius, the induction 
Table 4.1: Parameters of investigation for a single shocked interface case.

\begin{tabular}{|c|c|c|c|c|c|c|}
\hline$M_{s}$ & $\mathrm{~A}$ & $R_{s}$ & $R_{0}$ & $\mathrm{~m}$ & $\beta$ & \\
\hline 2.0 & 0.667 & 1.2 & 1.0 & 256 & $\infty$ & \\
2.0 & 0.667 & 1.2 & 1.0 & 64 & $\infty$ & Vary $m$ in hydrodynamics \\
2.0 & 0.667 & 1.2 & 1.0 & 16 & $\infty$ & \\
\hline 2.0 & 0.667 & 1.2 & 1.0 & 128 & 16 & \\
2.0 & 0.667 & 1.2 & 1.0 & 64 & 16 & Vary $m$ in MHD \\
2.0 & 0.667 & 1.2 & 1.0 & 16 & 16 & \\
\hline 2.0 & 0.667 & 1.2 & 1.0 & 256 & 128 & \\
2.0 & 0.667 & 1.2 & 1.0 & 256 & 32 & Vary $\beta$ in MHD \\
2.0 & 0.667 & 1.2 & 1.0 & 256 & 16 & \\
2.0 & 0.667 & 1.2 & 1.0 & 256 & 4 & \\
\hline
\end{tabular}

equation is satisfied identically and decoupled from the other equations.

To examine the solution of base state, we plot the density profiles of base state at time $t=0.0$ and $t=0.525$ in Fig. 4.2 . When the incident shock (IS) moves radially to the contact discontinuity $(\mathrm{CD})$, it undergoes refraction, resulting in a transmitted shock (TS) and a reflected shock (RS). This can be better illustrated in the density spacetime diagram. In the spacetime diagram, the distance will be displayed on the horizontal axis and the time on the vertical axis. In Fig. 4.3 , the vertical line (labeled as "CD") representing contact discontinuity sits stationary until the incident shock interacts with it. Then the incident shock bifurcates into a transmitted shock and a reflected shock (labeled as "TS" and "RS" respectively). The contact discontinuity moves because of the impulse due to the interaction with incident shock. It is represented by a curve between two shocks. While CD appears to be straight line in the spacetime diagram, it is not. The base velocity $\stackrel{\leftrightarrow}{r}_{r}$ of the CD is plotted in Fig 4.4. It shows that the CD decelerates as it gets close to the origin. We also note that at $t=0.525$, the transmitted shock is about to leave the domain. Therefore, we restrict all the results to a final time of $t=0.525$ to make sure all waves are in the computational domain. 


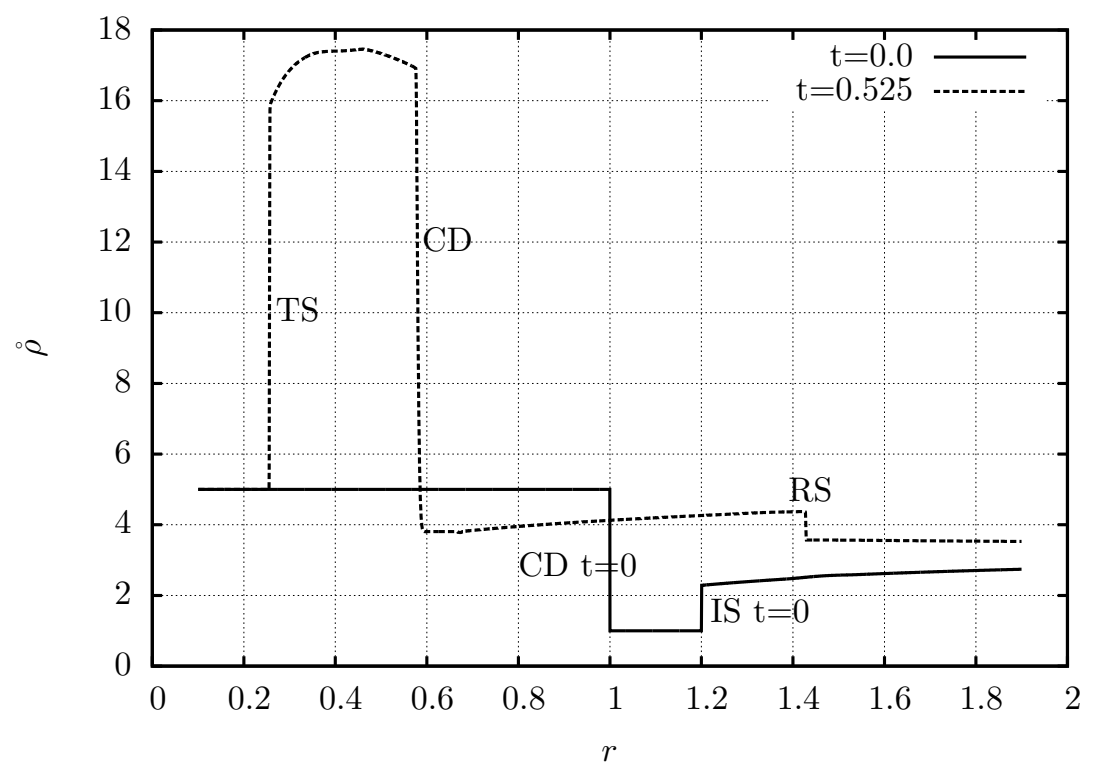

Figure 4.2: Density profiles of base state at $t=0.0$ and $t=0.525$. IS is the incident shock. TS is the transmitted shock. RS is the reflected shock. CD is the contact discontinuity.

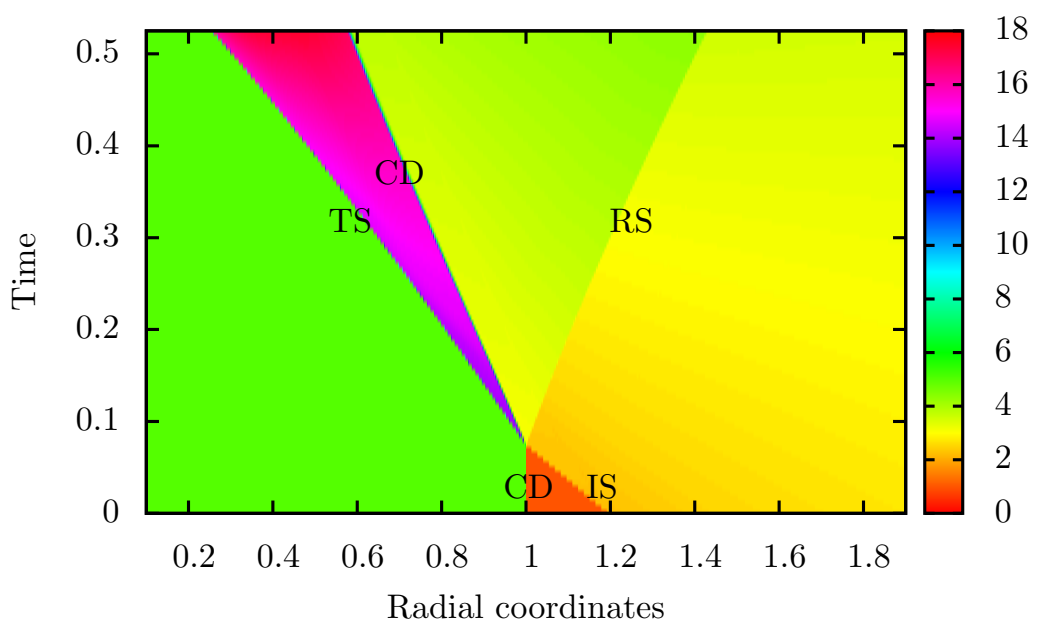

Figure 4.3: Spacetime diagram of density. IS is the incident shock. TS is the transmitted shock. RS is the reflected shock. CD is the contact discontinuity. 


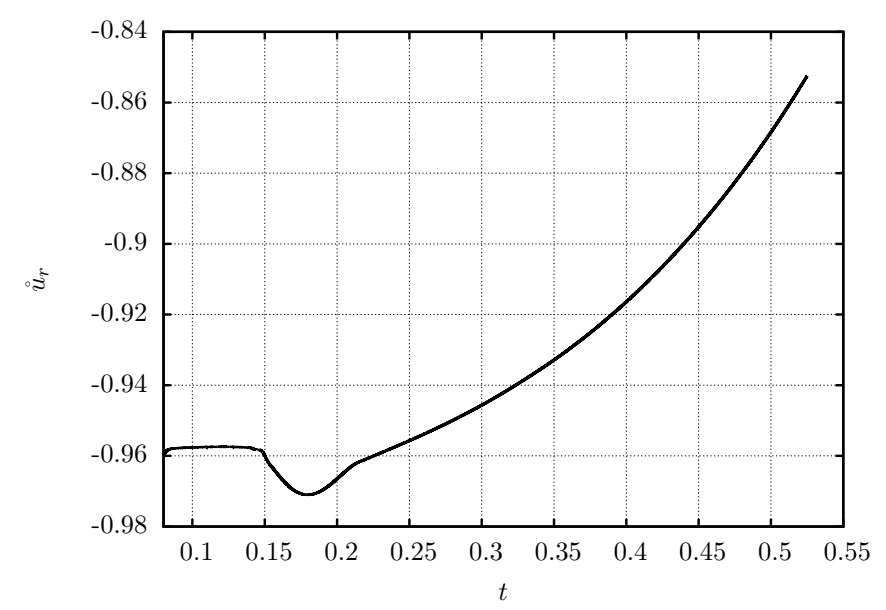

Figure 4.4: Time history of radial velocity $\stackrel{\varkappa}{r}_{r}$ at CD for gas dynamics.

\subsection{Solution Details for RMI in Hydrodynamics}

In this section, we present results for hydrodynamics $(\beta=\infty)$. In Fig. 4.5, we plot the scaled growth rate history in hydrodynamics for different perturbation wavenumbers $m=16,64,256$. We nondimensionalize both the time scale and growth rate, following Lombardini and Pullin[14]. We choose $R_{0} / a_{0} m$ as our reference time scale, where $a_{0}$ is the unshocked sound speed. The perturbation growth rate $\dot{h}$ is normalized by the theoretical growth rate $\dot{h}_{\infty}$ as

$$
\dot{h}_{\infty}=\frac{h(0)}{R_{0}} \Delta W\left(1+m A^{+}\right)
$$

where $\Delta W$ is the impulsive change in the interface velocity, $A^{+}$is the post-shock Atwood number and $h(0)$ is the initial perturbation amplitude and is chosen to be 1.0 .

For hydrodynamics, after the initial fast increase, similar to the Cartesian geometry, the perturbation growth rate oscillates around an asymptotic value. But as the interface approaches the origin, the perturbation growth rate starts to decrease exponentially. This is because the RTI begins to play an important role. For the 


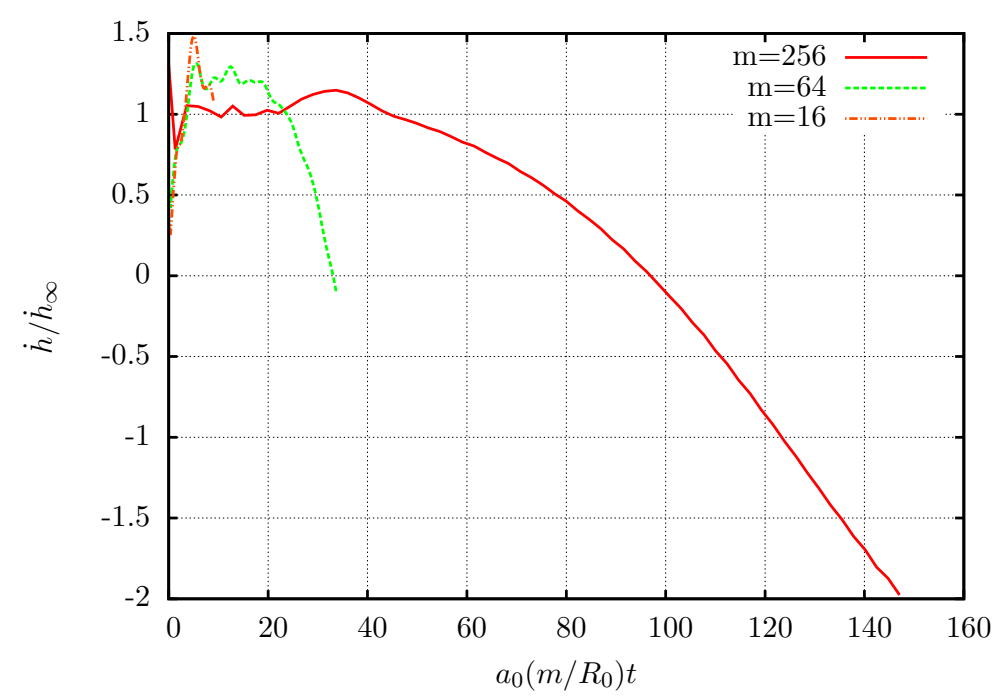

Figure 4.5: Time history of scaled growth rate in hydrodynamics for azimuthal perturbation wavenumbers $m=16,64,256$.

large wavenumber $(m=256)$, the onset of RTI is clear, but for a smaller wavenumber $(m=16)$, the demarcation between RMI and RTI is not apparent. This is because wavenumber $m$ is proportional to the development of growth rate. For smaller $m$. the time duration inspected is too short and the effect of RTI is insignificant.

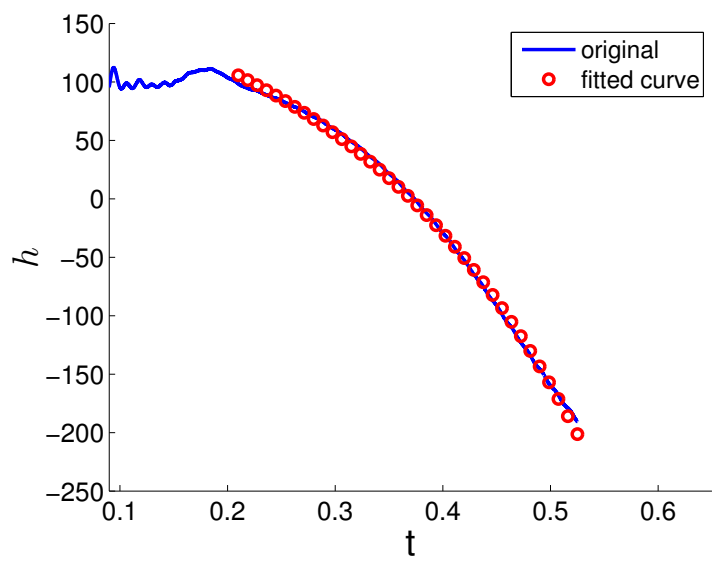

Figure 4.6: Time history of unscaled growth rate (solid line) and fitted RTI growth (circles) in hydrodynamics for azimuthal perturbation wavenumbers $m=256$.

To quantify the RTI, we perform a nonlinear curve fit to the RTI growth for 
$m=256,128,64$. All these curve fits are performed using the unscaled variables. The fitted equation is of the form $\left.2 \dot{h}\right|_{t_{0}}-\left.\dot{h}\right|_{t_{0}} \exp \left(\bar{\gamma}\left(t-t_{0}\right)\right)$, where $\bar{\gamma}$ is the growth rate of RTI, $t_{0}$ is the time of the onset of RTI and chosen by a visual inspection of the growth rate plot to be 0.21 . In Fig 4.6 , we plot the curve fit results of $m=256$. The fit results are tabulated in table 4.2. As expected, the fitted $\bar{\gamma}$ decreases as the wavenumber $m$ decreases.

Table 4.2: Exponential curve fit results of RTI for different wavenumber $m$

\begin{tabular}{|l|r|r|r|}
\hline$m$ & 256 & 128 & 64 \\
\hline $\bar{\gamma}$ & 4.33 & 3.50 & 2.52 \\
$\left.\dot{h}\right|_{t_{0}}$ & 105.58 & 70.60 & 38.22 \\
\hline
\end{tabular}

Fig. 4.7 plots perturbed $r$-component of velocity, perturbed $\theta$-component of velocity, perturbed $\theta$-component of momentum and perturbed $z$-component of vorticity for hydrodynamics of wavenumber $m=256$. After refraction, secondary waves interact with the interface, higher perturbation wavenumbers lead to smaller periods or higher frequency of oscillations observed in related solutions (Fig 4.7) and growth rate (Fig. 4.5). The vorticity $\hat{\omega}_{z}$ is a derived quantity defined by $\hat{\omega}_{z}=\frac{1}{r} \frac{\partial\left(r \hat{u}_{\theta}\right)}{\partial r}-\frac{1}{r} j m \hat{u}_{r}$. The peak of vorticity indicates the position of the vortex sheet (VS). The position of the VS coincides with the position of the interface in hydrodynamics. This can be seen again in the $\hat{u}_{\theta}$ plot where there is a huge gradient at the interface. Vorticity is generated baroclinically at the interface and is the driving mechanism behind the RMI, as discussed by Hawley and Zabusky[20] and Samtaney and Zabusky[21].

To study the case of smaller wavenumber $m=16$, Fig. 4.8 plots perturbed $r$ component of velocity, perturbed $\theta$-component of velocity, perturbed $\theta$-component of momentum and perturbed z-component of vorticity for hydrodynamics of wavenumber $m=16$. As we stated before, wavenumber $m$ is proportional to the development of growth rate. For $m=16$, from Fig. 4.5, the growth rate is in the early stage. Unlike the case of $m=256$, the perturbations at transmitted shock are large. 


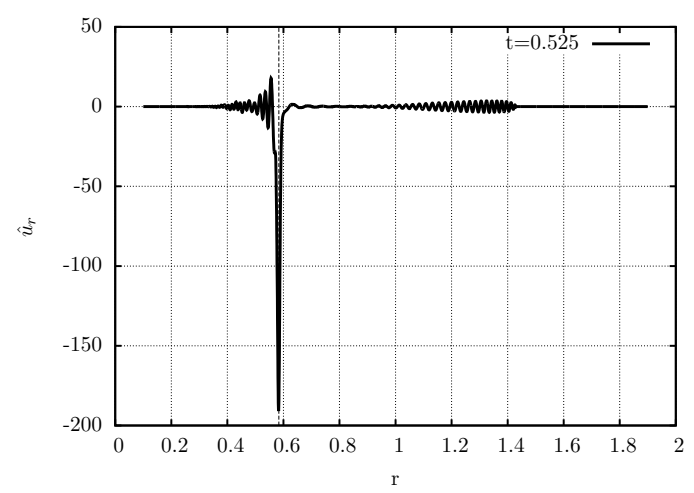

(a) $\hat{u}_{r}$ at $t=0.525$ for hydrodynamics

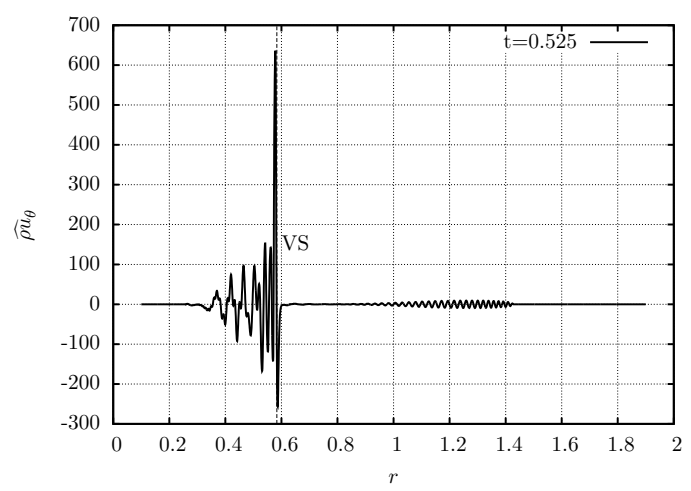

(c) $\widehat{\rho u}_{\theta}$ at $t=0.525$ for hydrodynamics

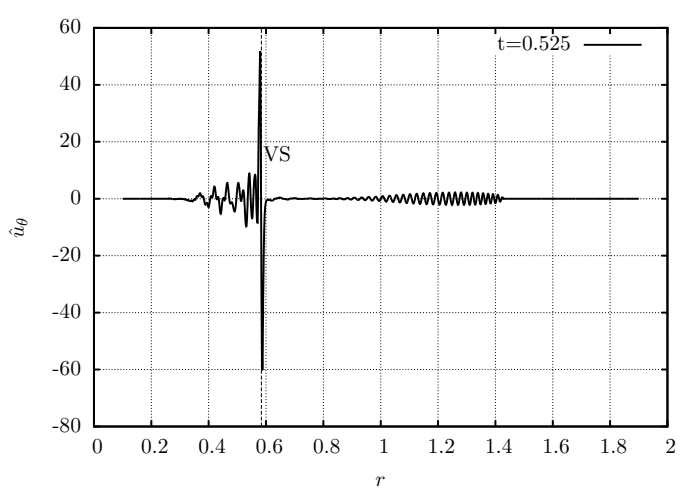

(b) $\hat{u}_{\theta}$ at $t=0.525$ for hydrodynamics

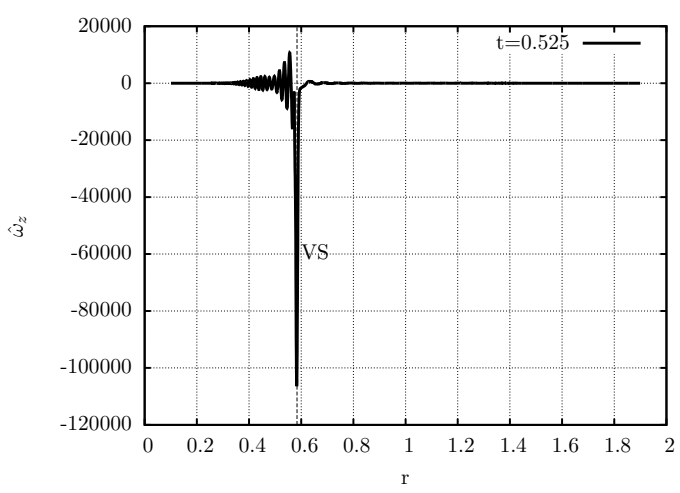

(d) $\hat{\omega}_{z}$ at $t=0.525$ for hydrodynamics

Figure 4.7: Profiles of (a) perturbed $r$-component of velocity for hydrodynamics. (b) perturbed $\theta$-component of velocity for hydrodynamics. (c) perturbed $\theta$-component of momentum for hydrodynamics. (d) perturbed $z$-component of vorticity for hydrodynamics. The perturbation wavenumber is $m=256$. 


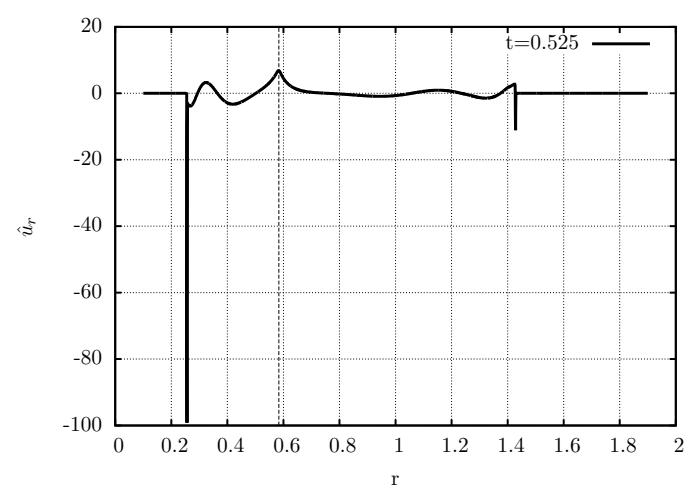

(a) $\hat{u}_{r}$ at $t=0.525$ for hydrodynamics

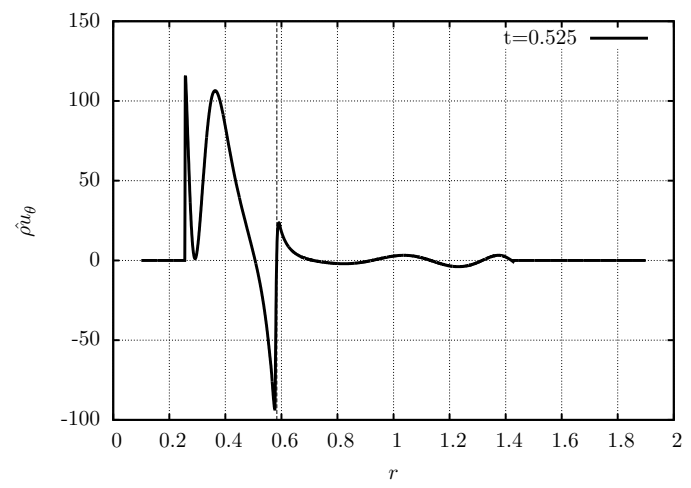

(c) $\widehat{\rho u}_{\theta}$ at $t=0.525$ for hydrodynamics

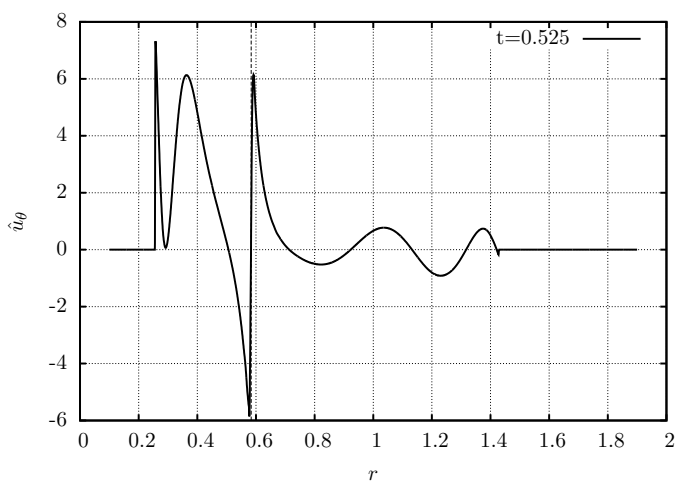

(b) $\hat{u}_{\theta}$ at $t=0.525$ for hydrodynamics

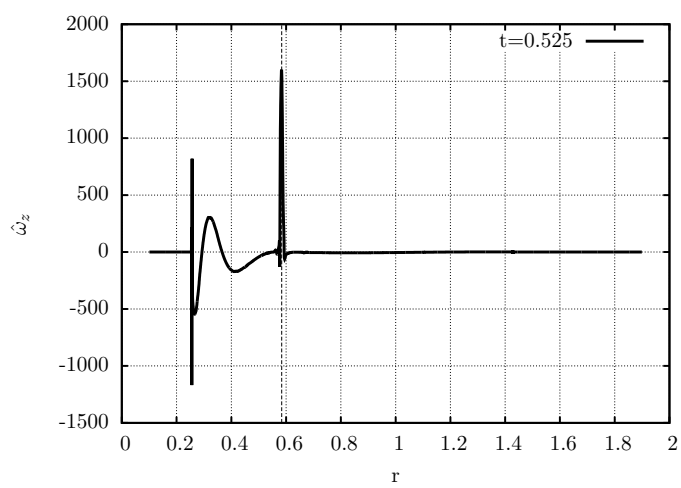

(d) $\hat{\omega}_{z}$ at $t=0.525$ for hydrodynamics

Figure 4.8: Profiles of (a) perturbed $r$-component of velocity for hydrodynamics. (b) perturbed $\theta$-component of velocity for hydrodynamics. (c) perturbed $\theta$-component of momentum for hydrodynamics. (d) perturbed $z$-component of vorticity for hydrodynamics. The perturbation wavenumber is $m=16$. 


\subsection{Solution Details for RMI in MHD}

We first choose the plasma beta $\beta=16$ at the interface $R_{0}=1.0$. In Fig. 4.9, we plot the scaled growth rate history in MHD for different perturbation wavenumbers $m=16,64,128,256$. For larger wavenumber $(m=256,128)$, initially the growth rate increases, but as the time goes on, the presence of magnetic field suppresses the growth rate. Finally, the interface growth rate oscillates around a small value below zero during the time investigated. This behavior is somewhat different from that observed in Cartesian geometry. In the Cartesian geometry, the magnetic field suppresses the growth rate at the interface and the growth rate oscillates around the asymptotic value zero. Therefore, it is conjectured that vorticity is being continually generated at the accelerating interface and at the same time being continuously removed from the interface by MHD waves. This probably results in a small residual growth rate. Also, the onset of RTI is not clearly observed in MHD. For smaller wavenumber $(m=64,16)$, the oscillations are not seen. Again, this is probably because the time durations investigated are not long enough.

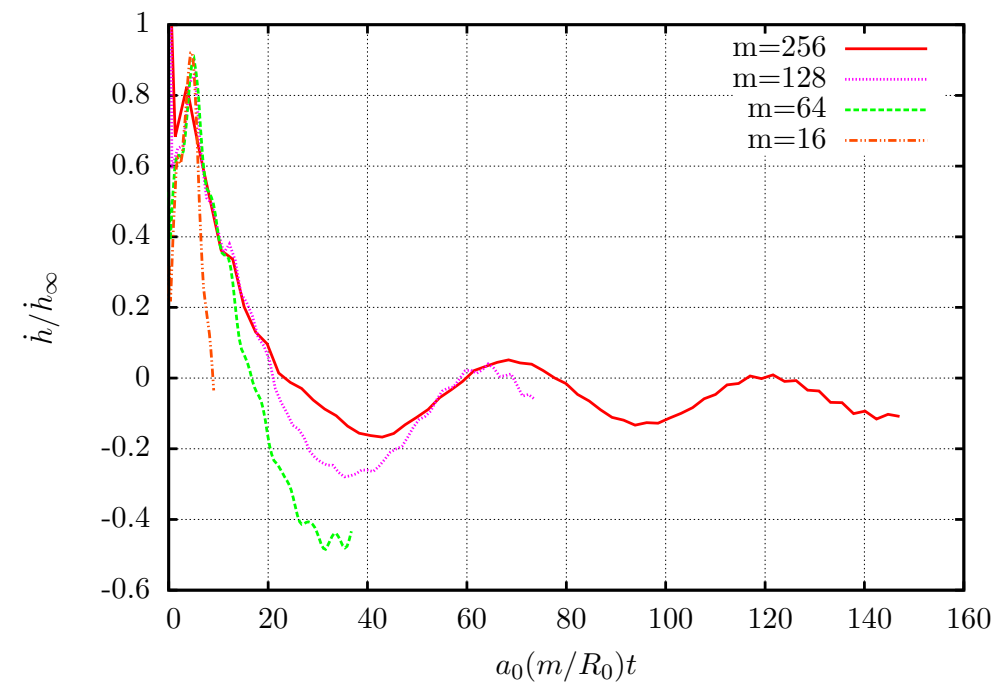

Figure 4.9: Time history of scaled growth rate in MHD for azimuthal perturbation wavenumbers $m=16,64,128,256$. The plasma beta is $\beta=16$ at the interface $R_{0}=1.0$. 


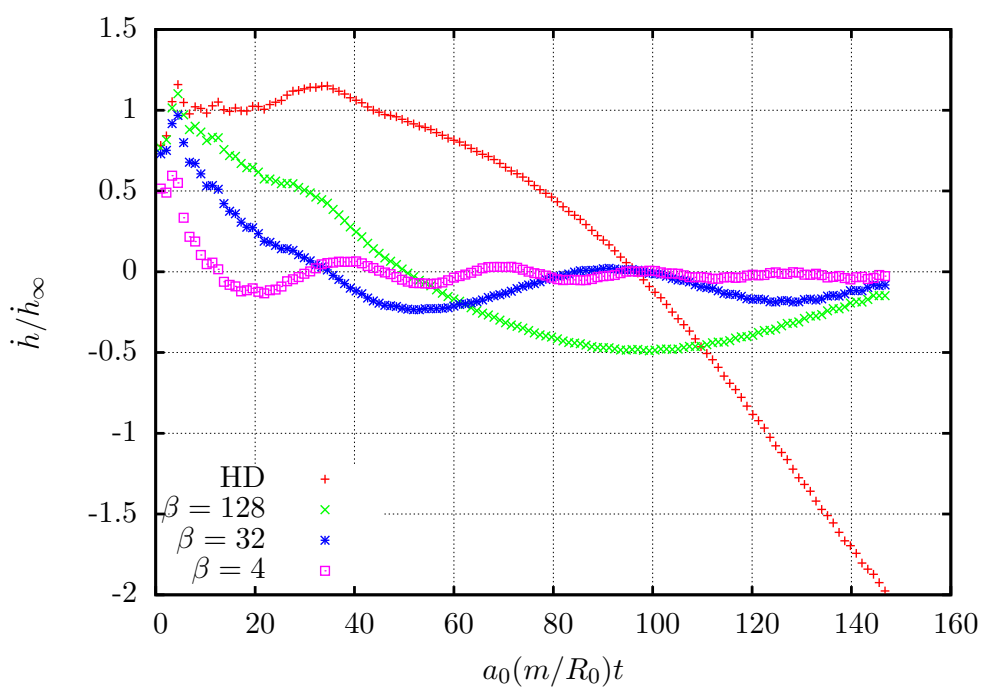

Figure 4.10: Time history of scaled growth rate in MHD for plasma beta $\beta=$ $\infty(H D), 128,32,4$ at the interface. The perturbation wavenumber is $m=256$.

To study the influence of different magnetic field strength, in Fig. 4.10, we plot the time history of scaled growth rate in MHD for plasma beta $\beta=\infty, 128,32,4$. The perturbation wavenumber is $m=256$. As expected, the stronger the magnetic field is, the faster the suppression occurs.

Fig. 4.11 plots perturbed $r$-component of velocity, perturbed $\theta$-component of velocity, perturbed $r$-component of magnetic field, perturbed $\theta$-component of magnetic field, perturbed z-component of vorticity and perturbed $z$-component of current for MHD of plasma beta $\beta=4$ and perturbation wavenumber $m=256$. The perturbed current $\hat{j}_{z}$ is another derived quantity defined by $\hat{j}_{z}=\frac{1}{r} \frac{\partial\left(r \hat{B}_{\theta}\right)}{\partial r}-\frac{1}{r} j m \hat{B}_{r}$. The peak of the current indicates the position of the Alfvén front (AF). Comparing the plot of vorticity and current, it is found that the positions of the VS coincide with the positions of the AF. In all the plots, the perturbations are large at the AF, but small at the interface. Vorticity is transported away from the interface by Alfvén fronts and this removes the driving force for the instability at the interface. 


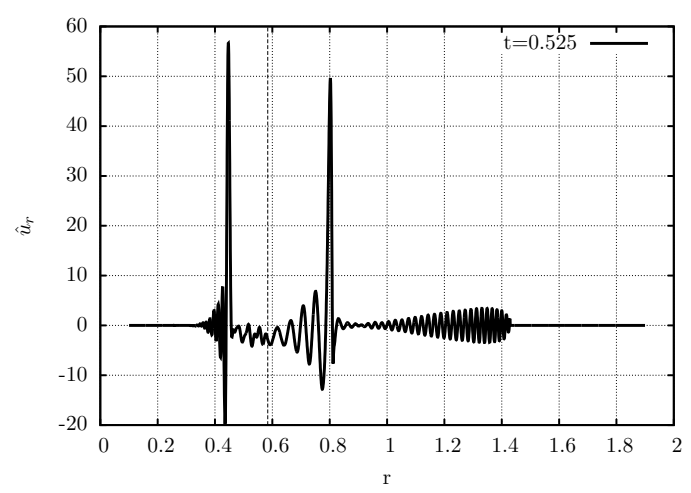

(a) $\hat{u}_{r}$ at $t=0.525$ for MHD

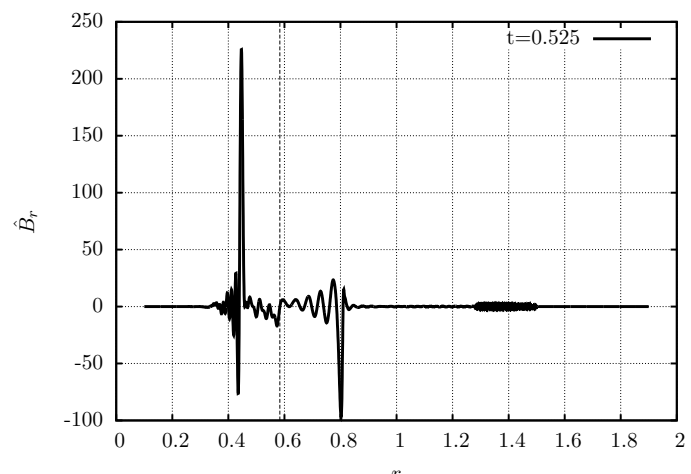

(c) $\hat{B}_{r}$ at $t=0.525$ for MHD

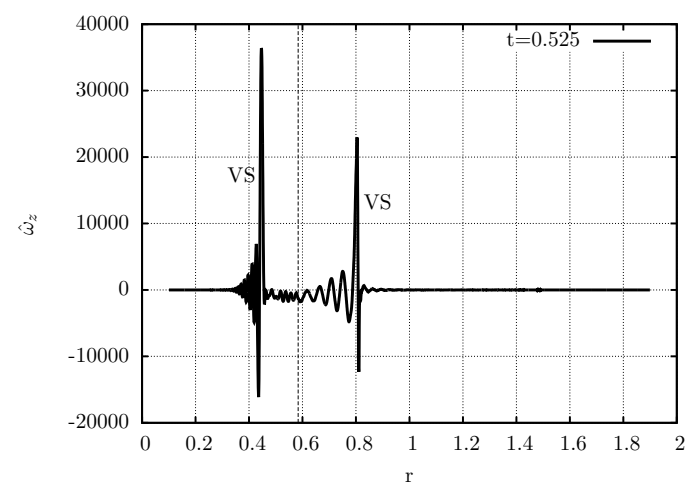

(e) $\hat{\omega}_{z}$ at $t=0.525$ for MHD

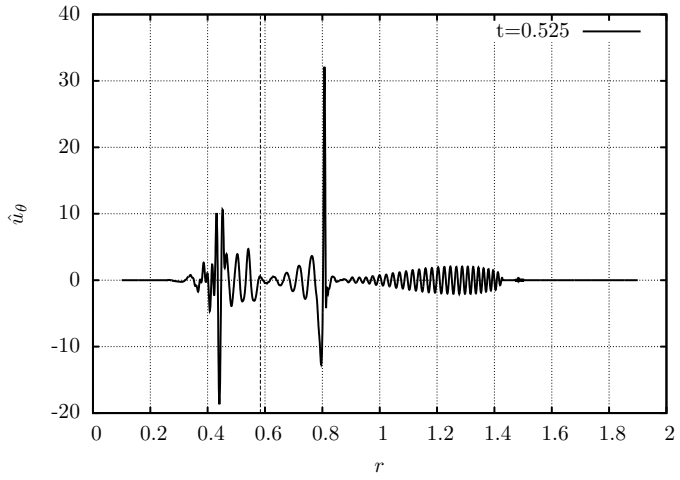

(b) $\hat{u}_{\theta}$ at $t=0.525$ for MHD

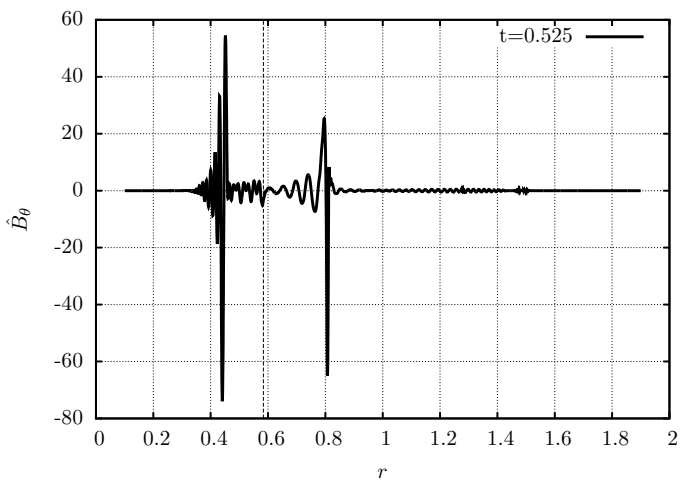

(d) $\hat{B}_{\theta}$ at $t=0.525$ for MHD

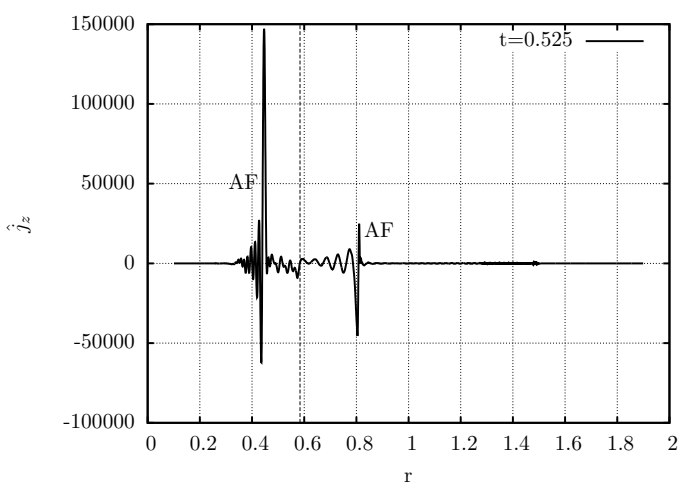

(f) $\hat{j}_{z}$ at $t=0.525$ for MHD

Figure 4.11: Profiles of (a) perturbed $r$-component of velocity for MHD. (b) perturbed $\theta$-component of velocity for MHD. (c) perturbed $r$-component of magnetic field for MHD. (d) perturbed $\theta$-component of magnetic field for MHD. (e) perturbed $z$-component of vorticity for MHD. (f) perturbed $z$-component of current for MHD. The perturbation wavenumber is $m=256$ and the plasma beta is $\beta=4$ at the interface $R_{0}=1.0$. 


\subsection{Comparison between hydrodynamics and MHD}

For perturbation wavenumber $m=256$, we compare the profiles of vorticity between hydrodynamics and MHD. For MHD, the plasma beta is $\beta=4$ at the interface $R_{0}=$ 1.0. From Fig. 4.12, the vorticity is concentrated at the interface for hydrodynamics. However, going from the hydrodynamics to the MHD, the vorticity bifurcates into two vortex sheets (marked as "VS"). The comparison of space time diagram of vorticity for hydrodynamics and MHD is plotted in Fig. 4.13. For hydrodynamics, the vorticity is primarily seen at the interface. Also in hydrodynamics, the vortex sheet changes sign eventually as the interface approaches the origin due to the RTI. For MHD, after the incidence shock interacts with the interface, the vortex sheet splits into two at the positions of the Alfvén fronts. The two vortex sheets will transport vorticity away from the interface, resulting in the decaying growth rate at the interface.

Fig. 4.14 plots the time history of circulation in the whole domain for hydrodynamics and MHD. In two dimensions, circulation is defined as $\Gamma=\oint_{l} \mathbf{u} \cdot d \mathbf{l}$. In our case, because $\stackrel{\leftrightarrow}{r}_{r}$ is constant and $\stackrel{\varkappa}{\theta}_{\theta}=0$, we get $\Gamma=\oint_{l} \mathbf{u} \cdot d \mathbf{l}=\oint_{l}(\stackrel{\mathbf{u}}{ }+\hat{\mathbf{u}}) \cdot d \mathbf{l}=$ $\oint_{l} \hat{\mathbf{u}} \cdot d \mathbf{l}=2 \int_{R_{l}}^{R_{r}} \hat{u}_{r} d r$. After the shock interacts with the interface, the same amount of circulation is generated baroclinically for both hydrodynamics and MHD. However, as time goes on, the RTI becomes dominant for hydrodynamics and the two curves depart. In MHD, most of the circulation is due to contribution of vorticity on the Alfvén fronts. 


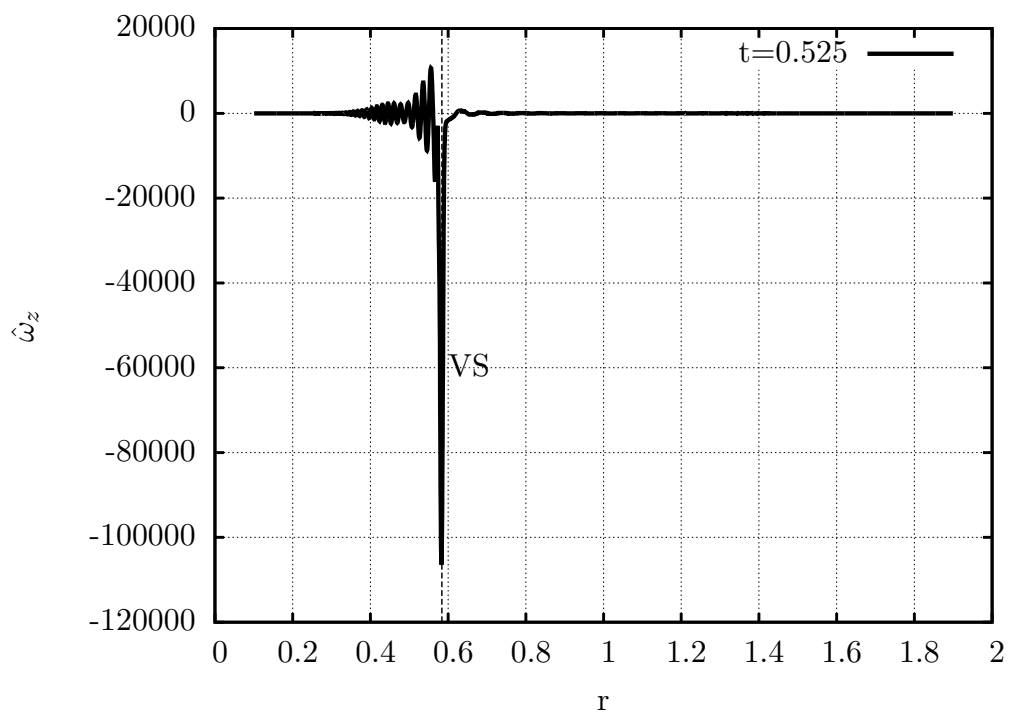

(a) $\hat{\omega}_{z}$ at $t=0.525$ for hydrodynamics

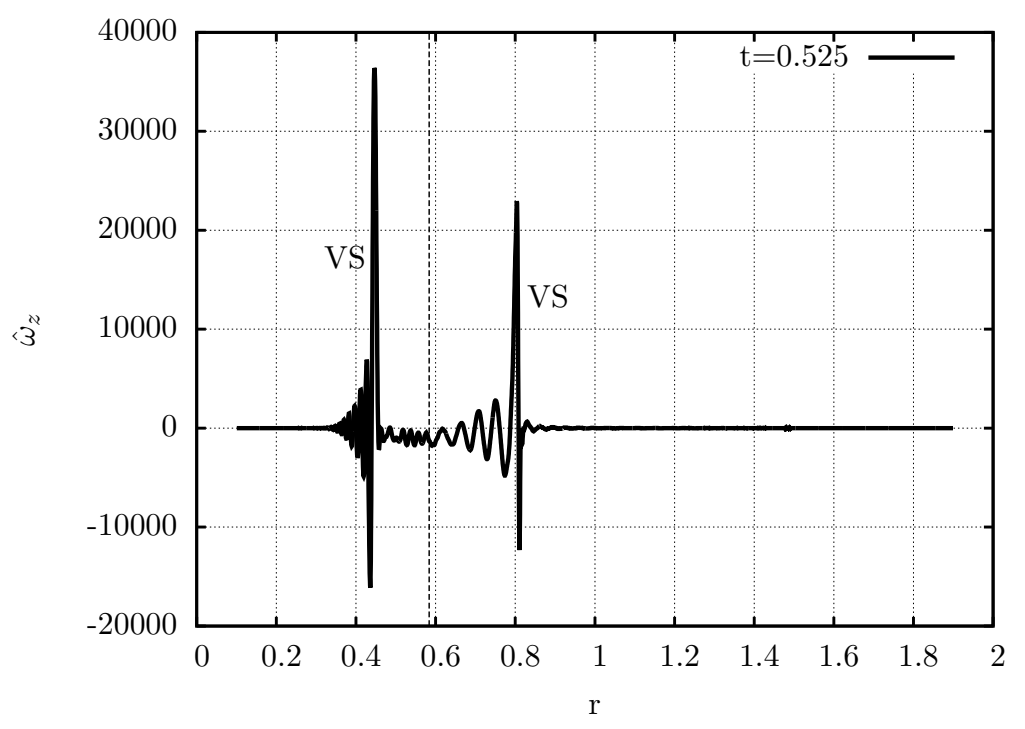

(b) $\hat{\omega}_{z}$ at $t=0.525$ for MHD

Figure 4.12: Profiles of (a) perturbed $z$-component of vorticity for hydrodynamics. (f) perturbed $z$-component of vorticity for MHD. The perturbation wavenumber is $m=256$ and the plasma beta is $\beta=4$ for MHD. 


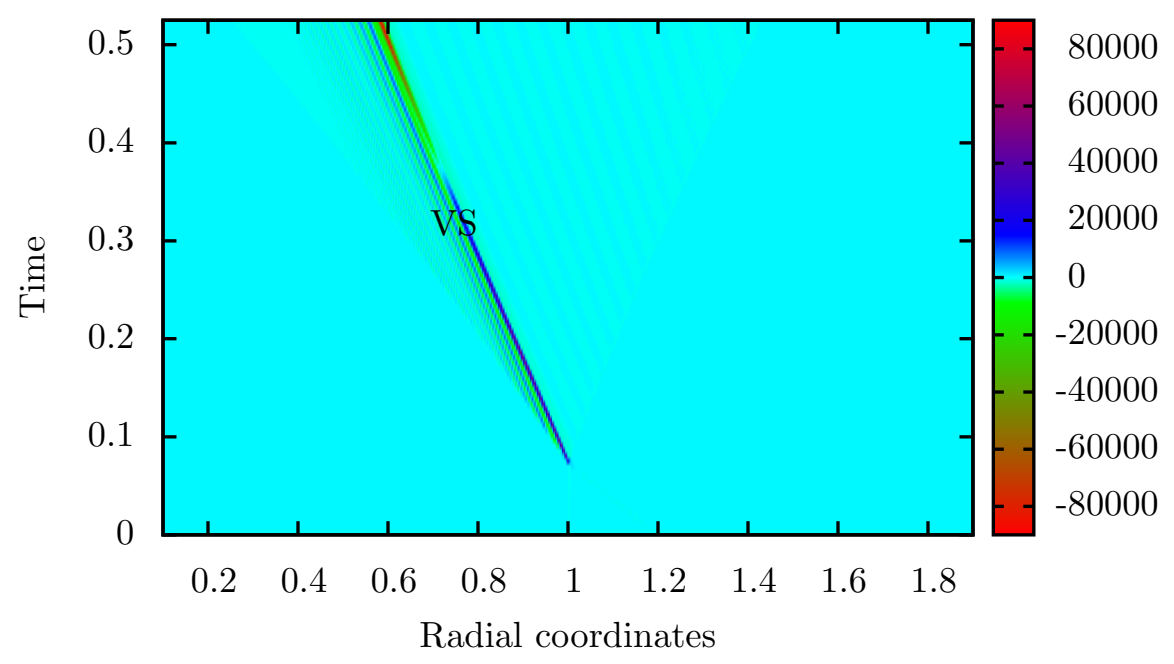

(a) Spacetime diagram of vorticity for hydrodynamics

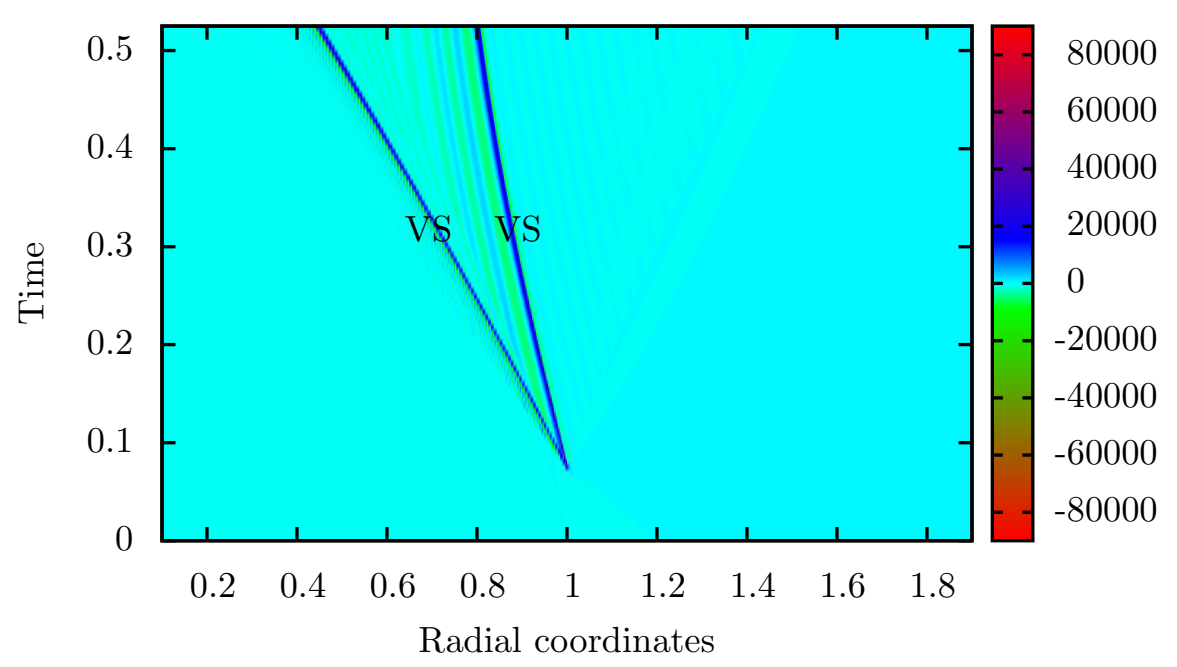

(b) Space time diagram of vorticity for MHD

Figure 4.13: (a) Spacetime diagram of vorticity for hydrodynamics. (f) Space time diagram of vorticity for MHD. The perturbation wavenumber is $m=256$ and the plasma beta is $\beta=4$ for MHD. 


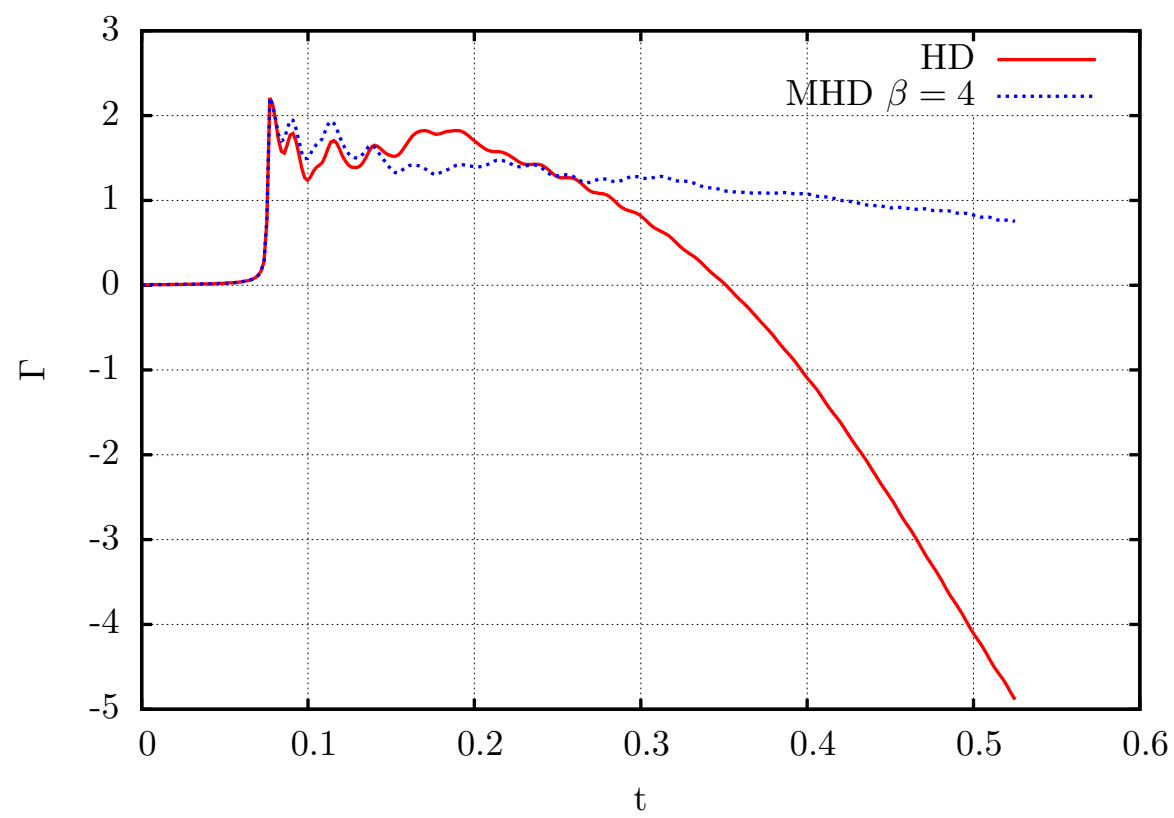

Figure 4.14: Time history of circulation in the whole domain for hydrodynamics and MHD. The perturbation wavenumber is $m=256$ and the plasma beta is $\beta=4$ for MHD. 


\section{Chapter 5}

\section{Conclusion}

\subsection{Summary}

We developed a numerical technique for solving the linearized MHD equations with a time-dependent base state with discontinuities. We perform the numerical linear stability analysis of positive Atwood number of interfaces with purely azimuthal perturbations interacting with a Chisnell-type imploding shock in cylindrical geometry. We conclude that

- For hydrodynamics, initially similar to Cartesian geometry, the baroclinic vorticity drives the Richtmyer-Meshkov instability growth, but then RayleighTaylor instability follows and dominates the growth rate. There is a phase change between RMI and RTI. The unscaled growth rate is proportional to wavenumber $m$. The larger the wavenumber, the larger the unscaled growth rate at the interface.

- For MHD, similar to Cartesian geometry, two Alfvén fronts transport vorticity away from the density interface and lead to the suppression of growth rate at the interface for sufficiently large wavenumbers and magnetic fields. The suppression of growth rate at the interface is significant for the case of perturbation wavenumber $m=256$ and plasma beta $\beta=4$. 


\section{$5.2 \quad$ Future Research Work}

The research work presented in this thesis can be further extended in the following aspects

- Perform a similar analysis in spherical geometry

- Consider axial perturbations in cylindrical geometry

- Simulate flows with multiple interfaces, like the cases in ICF

- Apply other magnetic field configurations

- Include negative Atwood ratio interfaces

- Compare linear stability results with nonlinear MHD simulations. 


\section{REFERENCES}

[1] J. D. Lindl, R. L. Mccrory, and E. M. Campbell. Progress toward ignition and burn propagation in inertial confinement fusion. Physics Today, 45(9):32-40, 1992.

[2] D. Arnett. The role of mixing in astrophysics. Astrophysical Journal Supplement Series, 127(2):213-217, 2000.

[3] R. D. Richtmyer. Taylor instability in shock acceleration of compressible fluids. Communications on Pure and Applied Mathematics, 13(2):297-319, 1960.

[4] E. E. Meshkov. Instability of the interface of two gases accelerated by a shock wave. Sov. Fluid Dyn., 4(5):101-104, 1969.

[5] Y. M. Yang, Q. A. Zhang, and D. H. Sharp. Small amplitude theory of RichtmyerMeshkov instability. Physics of Fluids, 6(5):1856-1873, 1994.

[6] R. Samtaney. Suppression of the Richtmyer-Meshkov instability in the presence of a magnetic field. Physics of Fluids, 15(8):L53-L56, 2003.

[7] V. Wheatley, R. Samtaney, and D. I. Pullin. The Richtmyer-Meshkov instability in magnetohydrodynamics. Physics of Fluids, 21(8), 2009.

[8] V. Wheatley, D. I. Pullin, and R. Samtaney. Regular shock refraction at an oblique planar density interface in magnetohydrodynamics. Journal of Fluid Mechanics, 522:179-214, 2005.

[9] V. Wheatley, D. I. Pullin, and R. Samtaney. Stability of an impulsively accelerated density interface in magnetohydrodynamics. Phys. Rev. Lett., 95:125002, 2005.

[10] T. Sano, K. Nishihara, C. Matsuoka, and T. Inoue. Magnetic field amplification associated with the Richtmyer-Meshkov instability. Astrophysical Journal, $758(2), 2012$. 
[11] M. Hohenberger, P. Y. Chang, G. Fiksel, J. P. Knauer, R. Betti, F. J. Marshall, D. D. Meyerhofer, F. H. Seguin, and R. D. Petrasso. Inertial confinement fusion implosions with imposed magnetic field compression using the OMEGAI Laser. Physics of Plasmas, 19(5), 2012.

[12] R. F. Chisnell. An analytic description of converging shock waves. Journal of Fluid Mechanics, 354:357-375, 1998.

[13] N. F. Ponchaut, H. G. Hornung, D. I. Pullin, and C. A. Mouton. On imploding cylindrical and spherical shock waves in a perfect gas. Journal of Fluid Mechanics, 560:103-122, 2006.

[14] M. Lombardini and D. I. Pullin. Small-amplitude perturbations in the threedimensional cylindrical Richtmyer-Meshkov instability. Physics of Fluids, 21(11), 2009.

[15] R. Samtaney. A method to simulate linear stability of impulsively accelerated density interfaces in ideal-MHD and gas dynamics. Journal of Computational Physics, 228(18):6773-6783, 2009.

[16] S. K. Godunov. Symmetric form of the equations of magnetohydrodynamics. Numerical Methods for Mechanics of Continuum Medium, 1:26, 1972.

[17] K. G. Powell, P. L. Roe, T. J. Linde, T. I. Gombosi, and D. L. De Zeeuw. A solution-adaptive upwind scheme for ideal magnetohydrodynamics. Journal of Computational Physics, 154(2):284-309, 1999.

[18] S. Falle, S. S. Komissarov, and P. Joarder. A multidimensional upwind scheme for magnetohydrodynamics. Monthly Notices of the Royal Astronomical Society, 297(1):265-277, 1998.

[19] R. Samtaney, P. Colella, T. J. Ligocki, D. F. Martin, and S. C. Jardin. An adaptive mesh semi-implicit conservative unsplit method for resistive MHD. In Journal of Physics: Conference Series, volume 16, page 40. IOP Publishing, 2005.

[20] J. F. Hawley and N. J. Zabusky. Vortex paradigm for shock-accelerated densitystratified interfaces. Physical Review Letters, 63:1241-1244, 1989.

[21] R. Samtaney and N. J. Zabusky. Circulation deposition on shock-accelerated planar and curved density-stratified interfaces: models and scaling laws. Journal of Fluid Mechanics, 269:45-78, 1994. 
APPENDICES 


\section{A Generating converging shock}

\section{waves in cylindrical geometry}

\section{A.1 Governing equations of Chisnell-type shock}

Chisnell [12] described the process to generate strong shock waves in cylindrical geometry. We follow Chisnell's process and then extend it to general shock waves.

Under the assumptions of symmetric adiabatic flow and ideal gas, the governing equations behind the shock are

$$
\begin{aligned}
\rho_{t}+u \rho_{r}+\rho r^{1-s}\left(u r^{s-1}\right)_{r} & =0 \\
u_{t}+u u_{r}+\frac{1}{\rho} p_{r} & =0 \\
\left(\frac{\partial}{\partial t}+u \frac{\partial}{\partial r}\right)\left(\ln \left(p / \rho^{\gamma}\right)\right) & =0 .
\end{aligned}
$$

Where $\gamma$ is the ratio of specific heats. $s$ equals 2 and 3 for cylindrical geometry and spherical geometry, respectively. $r$ is the distance from origin. $t$ is the time, and we assume the time that shock reach origin is 0 , therefore, the time $t$ is negative during our study. $\rho$ is density, $u$ is velocity and $p$ is pressure.

Chisnell then introduced three non-dimensional variables $G, V$ and $Z$

$$
\rho=\rho_{0} G, \quad u=\frac{r}{t} V, \quad c^{2}=\frac{r^{2}}{t^{2}} Z
$$

Where $c$ is the sound speed defined as $c^{2}=\gamma p / \rho$. The subscript 0 means the states 
ahead of shock, which is assumed to be constant. The gas is assumed to be at rest ahead of shock, i.e. $u_{0}=0$. Substitute the definition of $G, V$ and $Z$ into the governing Eqn. A.1.

$$
\begin{array}{r}
t G_{t}+V r G_{r}+G r V_{r}=-s V G, \\
t V_{t}+V r V_{r}+\frac{1}{\gamma} \frac{Z}{G} r G_{r}+\frac{1}{\gamma} r Z_{r}=V-V^{2}-\frac{2 Z}{\gamma}, \\
t \frac{Z_{t}}{Z}+r V \frac{Z_{r}}{Z}-\frac{\gamma-1}{G}\left(t G_{t}+V r G_{r}\right)=2-2 V .
\end{array}
$$

The self-similar variable $\xi$ is defined as

$$
\xi=r / R(t)
$$

Where $R(t)$ is the distance of shock from the origin and is a function of time $t$. Thus $G, V, Z$ are all function of $\xi$ alone. In order for the self-similar solutions to exist, the following equation has to be satisfied

$$
\frac{t \dot{R}}{R}=\alpha
$$

Where $\alpha$ is the self-similar coefficient.

Represent $G, V$ and $Z$ as a function of $\xi$

$$
\begin{array}{r}
\xi V^{\prime}+(V-\alpha) \xi \frac{G^{\prime}}{G}=-s V, \\
(V-\alpha) \xi V^{\prime}+\frac{Z}{\gamma} \xi \frac{G^{\prime}}{G}+\frac{1}{\gamma} \xi Z^{\prime}=V-V^{2}-\frac{2 Z}{\gamma}, \\
(\gamma-1) Z \xi \frac{G^{\prime}}{G}-\xi Z^{\prime}=-\frac{2 Z(1-V)}{V-\alpha}
\end{array}
$$


Further simplify

$$
\xi V^{\prime}(\xi)=\frac{\Delta_{1}}{\Delta}, \quad \xi \frac{G^{\prime}(\xi)}{G(\xi)}=\frac{\Delta_{2}}{\Delta}, \quad \xi Z^{\prime}(\xi)=\frac{\Delta_{3}}{\Delta}
$$

Where

$$
\begin{aligned}
& \Delta=-Z+(V-\alpha)^{2} \\
& \Delta_{1}=-\Delta\left\{s V-\frac{2(1-\alpha)}{\gamma}\right\}-(\alpha-V) Q(V), \\
& \Delta_{2}=\frac{2(1-\alpha)}{\gamma(\alpha-V)} \Delta-Q(V), \\
& \Delta_{3}=\frac{Z}{V-\alpha}\left[2 \Delta\left\{\alpha-V+\frac{1-\alpha}{\gamma}\right\}+(\gamma-1)(\alpha-V) Q(V)\right], \\
& Q(V)=s V(V-\alpha)+\frac{2(1-\alpha)}{\gamma}(\alpha-V)-V(V-1) .
\end{aligned}
$$

Next, the boundary condition at $\xi=1$ will be given.

According to the Rankine-Hugoniot jump conditions, the states just behind the shock are

$$
\begin{aligned}
\frac{\rho_{s}}{\rho_{0}} & =\frac{(\gamma+1) M^{2}}{(\gamma-1) M^{2}+2}, \quad u_{s}=-c_{0} M\left[\frac{2\left(M^{2}-1\right)}{(\gamma+1) M^{2}}\right], \quad \frac{p_{s}}{p_{0}}=1+2 \frac{\gamma}{\gamma+1}\left(M^{2}-1\right) . \\
\frac{c_{s}}{c_{0}} & =\sqrt{\left[\frac{2(\gamma-1)\left(\gamma M^{2}+1\right)}{(\gamma+1)^{2}}\right]\left[\frac{M^{2}-1}{M^{2}}+1\right]}
\end{aligned}
$$

Where the subscript $s$ means states just behind the shock. $M$ is the Mach number and is defined by $M=\dot{R}(t) / c_{0}$.

Using the definition of $G, V$ and $Z$ from Eqn. A.2, we get

$$
G_{s}=\frac{\rho_{s}}{\rho_{0}} \quad V_{s}=\frac{t_{I} u_{s}}{r_{0}} \quad Z_{s}=\frac{c_{s}^{2} t_{I}^{2}}{r_{0}^{2}}
$$

Where $t_{I}$ is the initial time, which is given by $t_{I}=-\alpha r_{0} / M c_{0} . r_{0}$ is the initial 
shock radius.

To sum up, the ordinary differential equations we are trying to solve are Eqn. A.7) along with the boundary conditions $(\mathrm{A} .10)$. The problem left is how to specify the self-similar coefficient $\alpha$.

\section{A.2 Determining self-similar coefficient $\alpha$}

From Eqn. A.7), we can easily get

$$
\frac{1}{Z} \frac{d Z}{d V}=\frac{2 \Delta(\alpha-V+(1-\alpha) / \gamma)+(\gamma-1)(\alpha-V) Q}{\Delta(s V-2(1-\alpha) / \gamma)(\alpha-V)+(\alpha-V)^{2} Q}
$$

After some singular points analysis, Chisnell suggested to substitute the trial function $Z_{T}$ into Eqn. A.11.

$$
Z_{T}=\lambda V^{2}, \quad \lambda=\left(\alpha / V_{0}-1\right)^{2}
$$

Where $V_{0}$ satisfy $Q\left(V_{0}\right)=0$ and $Z_{0}$ is defined as $\Delta\left(V_{0}, Z_{0}\right)=0$. The benefit of using trial function $Z_{T}$ is that an analytic solution can be obtained while still having a good approximation of the original solution. Eqn. A.11 now is

$$
\frac{1}{Z} \frac{d Z}{d V}-\frac{\eta}{\alpha-V}=\frac{2}{V}+\frac{B}{V+q}
$$

Where

$$
\begin{array}{r}
B=\eta+(s-1) \frac{2 \lambda+\gamma-1}{1-s \lambda} \\
q=\frac{-\alpha}{V_{0}(1-s \lambda)} \\
\eta^{-1}=\frac{s \alpha \gamma}{2(1-\alpha)}-1
\end{array}
$$


66

Table A.1: Comparison between Algorithm 1 and Chisnell's Paper.

\begin{tabular}{|ccccc|}
\hline & Chisnell's Paper & \multicolumn{3}{c}{ Algorithm $\mathbf{1}$} \\
$\gamma$ & & Mach $=100$ & Mach $=3$ & Mach $=2$ \\
\hline 1.2 & 0.86117 & 0.86115 & 0.84347 & 0.84036 \\
1.4 & 0.83532 & 0.83531 & 0.82860 & 0.83023 \\
$5 / 3$ & 0.81562 & 0.81562 & 0.81477 & 0.81990 \\
2 & 0.80011 & 0.80011 & 0.80266 & 0.81026 \\
3 & 0.77566 & 0.77566 & 0.78193 & 0.79274 \\
\hline
\end{tabular}

Integrate Eqn. A.13) from $V_{0}$ to $V_{s}$ gives

$$
\frac{Z_{s}}{Z_{0}}=\left(\frac{\alpha-V_{0}}{\alpha-V_{s}}\right)^{\eta}\left(\frac{V_{s}}{V_{0}}\right)^{2}\left(\frac{V_{s}+q}{V_{0}+q}\right)^{B}
$$

Rewrite $Q\left(V_{0}\right)=0$

$$
\frac{(s-1) \alpha}{1-\alpha}=\frac{1}{1-V_{0} / \alpha}+\frac{2}{\gamma V_{0} / \alpha}
$$

Using Eqn. A.9), A.10), A.15) and A.16), given any Mach number, we can obtain the self-similar coefficient $\alpha$ numerically. $V_{0} / \alpha$ is used as the Picard iteration parameter. The algorithm is described

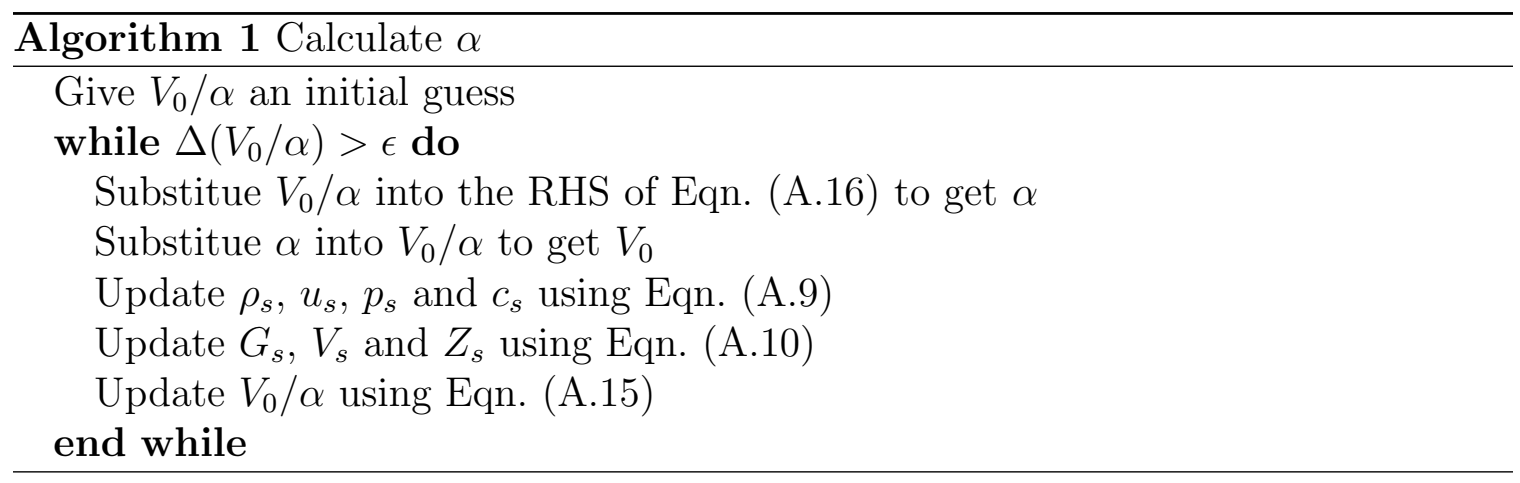

The $\alpha$ in Chisnell's paper can be used as an initial guess. The damping method can be used if the algorithm does not converge.

In table A.1, we compare the $\alpha$ computed from algorithm 1 and $\alpha$ listed in Chisnell's paper. Please note that Chisnell's results were got under the assumption that the shock is strong $($ Mach $=\infty)$.

The $\alpha$ of Mach 100 agrees with the results in Chisnell's paper which assumed 
strong shock. However, for the lower Mach number, there are some discrepancies. In this thesis, as we are interested in more general shock, we adopt $\alpha$ obtained from algorithm 1 and then solve Eqn. A.7 to get the initial condition. 


\section{B Papers Submitted and Under Preparation}

- S. Gao and R. Samtaney, "Linear Simulations of the Cylindrical Richtmyer-Meshkov Instability in Hydrodynamics and MHD", Submitted to 29th International Symposium on Shock Wavese, July 14-19, 2013, Madison, Wisconsin. Also presented on poster for IAMCS in KAUST on May 12, 2013.

- S. Gao and R. Samtaney, "Linear Stability Analysis of the Cylindrical RichtmyerMeshkov Instability in Hydrodynamics and MHD", Physics of Fluids, under preparation. 